\title{
DPM, a fast, accurate Monte Carlo code optimized for photon and electron radiotherapy treatment planning dose calculations
}

\author{
Josep Sempau $\dagger \ddagger$, Scott J Wilderman $\dagger$ and Alex F Bielajew $\dagger$ \\ $\dagger$ Department of Nuclear Engineering and Radiological Sciences, The University of Michigan, \\ Ann Arbor, MI, USA \\ \$ Institut de Tècniques Energètiques, Universitat Politècnica de Catalunya, Diagonal 647, \\ 08028 Barcelona, Spain
}

Received 29 February 2000

\begin{abstract}
A new Monte Carlo (MC) algorithm, the 'dose planning method' (DPM), and its associated computer program for simulating the transport of electrons and photons in radiotherapy class problems employing primary electron beams, is presented. DPM is intended to be a highaccuracy MC alternative to the current generation of treatment planning codes which rely on analytical algorithms based on an approximate solution of the photon/electron Boltzmann transport equation. For primary electron beams, DPM is capable of computing 3D dose distributions (in $1 \mathrm{~mm}^{3}$ voxels) which agree to within $1 \%$ in dose maximum with widely used and exhaustively benchmarked general-purpose public-domain MC codes in only a fraction of the CPU time. A representative problem, the simulation of 1 million $10 \mathrm{MeV}$ electrons impinging upon a water phantom of $128^{3}$ voxels of $1 \mathrm{~mm}$ on a side, can be performed by DPM in roughly 3 min on a modern desktop workstation. DPM achieves this performance by employing transport mechanics and electron multiple scattering distribution functions which have been derived to permit long transport steps (of the order of $5 \mathrm{~mm}$ ) which can cross heterogeneity boundaries. The underlying algorithm is a 'mixed' class simulation scheme, with differential cross sections for hard inelastic collisions and bremsstrahlung events described in an approximate manner to simplify their sampling. The continuous energy loss approximation is employed for energy losses below some predefined thresholds, and photon transport (including Compton, photoelectric absorption and pair production) is simulated in an analogue manner. The $\delta$-scattering method (Woodcock tracking) is adopted to minimize the computational costs of transporting photons across voxels.
\end{abstract}

\section{Introduction}

Several researchers have recently suggested that Monte Carlo (MC) based systems will soon become the dominant vehicles for dose computation in radiotherapy treatment planning (Bielajew 1994a, 1997, Mohan 1997, Hartmann-Siantar et al 1997). The superior accuracy of the MC method, which converges to results which are exact to the degree to which physical parameters are known, over that of deterministic models has long been well established. Public domain codes such as EGS4 (Nelson et al 1985, Bielajew et al 1994), ITS (Halbeib 1989, Halbeib et al 1992), MCNP (Briesmeister 1993), and PENELOPE (Baró et al 1995, Salvat et al 1996, Sempau et al 1997) have all been extensively benchmarked against experimental data for a wide range of materials and energies. EGS4 in particular has been thoroughly tested in the specific region of dosimetric interest (Rogers and Bielajew 1989b, 1990), and is widely accepted as a computational standard for radiotherapy dose 
calculations. Further, the near equivalence of the prevalent MC codes has also been established (Rogers and Bielajew 1989a, Andreo 1991), and their differences shown to be of little significance in the radiotherapy dose calculation problem†.

By contrast, the deterministic algorithms currently used for calculating electron dose in treatment planning systems rely on analytic approximations to the solution of the transport equation which fail to adhere to their limiting conditions in certain radiotherapy applications. Errors up to 50\% for electron beams (Cygler et al 1987) and up to 30\% for problems involving photon and electron transport near inhomogeneities (Ma et al 1999) have been reported. These discrepancies arise because the deterministic methods are based on approximate analytical solutions of the transport problem in semi-infinite media which are then modified semiempirically to account for inhomogeneities. Such methods are often not adequate for treatment planning computations in which interfaces between materials with large differences in density and/or atomic numbers (e.g. soft tissue, bone and air) play an important role. MC based techniques, on the other hand, are capable of modelling heterogeneities with a fine granularity.

Thus far, the impediment to the widespread implementation of MC based methods for dose computation has been that, even with continuing advances in computer architecture and clock speed, the currently available codes are quite slow. The practical requirement imposed by clinical radiotherapy treatment planning systems is to provide dose distributions of sufficient accuracy ( $2-3 \%$ of the dose maximum) within a time of practical clinical relevance ( $\lesssim 5 \mathrm{~min}$ ) and with a modest investment in computer hardware. Though most MC programs are sufficiently fast for simulating dose deposition in homogeneous media and simple geometries, radiotherapy applications involve numerous variations of material and density over small distances. Patient geometry is usually simulated as a map of densities over a large number $\left(128^{3}\right)$ of relatively small $(\sim 1-4 \mathrm{~mm})$ parallelepipeds (voxels), obtained from computed tomography (CT) scans. Currently, Monte Carlo simulation of absorbed dose for such large-scale problems is feasible only when employing computer resources of a scale not generally available in medical centres (Hartmann-Siantar et al 1995, 1997, Ma et al 1999).

Recently, Keall and Hoban (1996), Neuenschwander and Born (1992), Neuenschwander et al (1995), Kawrakow et al (1996) and the PEREGRINE code (Hartmann-Siantar et al 1995,1997 ) have attempted to surmount the CPU constraint by significantly modifying the basic MC electron transport algorithm. These new methods rely on some combination of simplifying the physics to different degrees of accuracy; reusing all or parts of particle histories; and/or implementing parallel processing (requiring a significant investment in hardware). In this work we present a new MC algorithm, the 'dose planning method' (DPM) for the simulation of coupled electron-photon transport in radiotherapy treatment planning without reliance on these limiting approximations and requirements.

DPM employs the standard condensed history model for electron transport, and falls into what has been called by Berger (1963) a 'mixed' scheme for the treatment of energy losses, treating large energy transfer collisions in an analogue sense and using the continuous slowing down approximation (CSDA) to model small-loss collisions. Gains in performance derive from a series of significant enhancements to the algorithm for transporting particles from point to point (the 'transport mechanics') and corresponding reformulation of the distribution functions describing the physics, as described below.

The first modification involves the employment and refinement of a new step size independent multiple-scattering theory (Kawrakow and Bielajew 1998b). Our method

$\dagger$ The comparisons performed by Rogers and Bielajew employed the ETRAN code (Berger 1963, Seltzer 1989, 1991) from which the electron transport physics (with some subtle modifications) for ITS and MCNP was derived. 
is a robust implementation of the Lewis (1950) formulation of Goudsmit-Saunderson theory (Goudsmit and Saunderson 1940a, b). Both provide exact solutions for the angular distribution of an electron traversing a given distance. However, the Lewis formulation allows the cross section to vary along the path according to the CSDA energy-loss model. The underlying cross section used here is the screened Rutherford cross section with the Molière screening factor (Molière 1947), after that implemented in Molière's small-angle multiple-scattering theory (Molière 1948) and employed in the EGS4 code with a correction for large angle suggested by Bethe (1953). From the validity of the EGS4 code for radiotherapy applications, we infer that the use of this form is sufficiently accurate. In addition to being derived under an exact framework, because it can be recast into a form independent of energy, the Kawrakow-Bielajew multiple scattering formulation provides a vehicle which can be exploited to permit transport across inhomogeneities, as discussed later. Use of the KawrakowBielajew distribution provides one other advantage. Larsen (1992) has demonstrated that the accuracy of a transport simulation scheme for charged particles is dominated by the faithfulness of the multiple-scattering theory it employs. In the limit of small electron step size, the correct solution to the transport equation is guaranteed if and only if the multiple-scattering theory faithfully reproduces the discrete single-scattering distributions. To our knowledge, the Kawrakow-Bielajew formalism yields the only purely multiple-scattering distribution function which is correct in the multiple-, plural- and single-scattering regimes. Thus, DPM is guaranteed to always converge to a correct solution as the step size is reduced.

The second major innovation introduced by DPM lies in the use of new transport mechanics, i.e. the algorithm for moving charged particles from point-to-point in media given the composition of the material traversed, the length of the step and the multiple-scattering angle. Larsen's work suggests that the schemes employed in current general-purpose codes are not optimal, and that a measure of the quality of a transport mechanism is measured by how quickly it converges to the small step size limit. Transport schemes can be characterized also by their adherence to the exact spatial-angular moments first reported by Lewis (1950), and a new scheme with high-order convergence has been reported recently (Kawrakow and Bielajew 1998a). The implementation of this new method is computationally and algorithmically demanding, however, and so DPM has adopted the 'random hinge' scheme $\dagger$ employed in PENELOPE. This algorithm has been shown to be almost as accurate as the KawrakowBielajew (1998a) transport mechanics in preserving the basic Lewis moments, but has a much simpler implementation. Recent work of Larsen $\ddagger$ and of Bielajew and Salvat (2000) demonstrates that higher-order convergent schemes exist, but they were not studied due to algorithmic complexity.

A third new technique introduced by DPM is the use of large electron transport steps, in which many voxels may be traversed before sampling a multiple scattering angle. This is made possible because of the stability of the random hinge algorithm across heterogeneities, the accuracy of the Kawrakow-Bielajew distribution, and because the multiple-scattering angle, when the step size is suitably scaled in terms of energy and scattering in the medium (as described below), is very nearly independent of atomic number. This feature of multiple scattering distributions has been shown to be rigorously true in small-angle theory (Bothe 1921a,b, Wentzel 1922, Molière 1948, Bielajew 1994b). We thus assume that the small residual dependences on the media of both the scattering distributions and of the random hinge Lewis moments can be safely ignored for radiotherapy-class problems, and relatively large steps (of the order of $5 \mathrm{~mm}$ ) can be employed.

\footnotetext{
$\dagger$ We are grateful to Dr Ronald Kensek of Sandia National Laboratories for this colourful nomenclature. $\ddagger$ We are grateful to Dr Ed Larsen of the University of Michigan for providing us with this information ahead of publication.
} 
Because the differential cross section is a fairly strong function of energy and there is significant energy loss over the long steps taken in DPM, a fourth modification has been introduced which scales the step sizes by the number of (material and energy dependent) transport mean free paths traversed. This preserves the total amount of scattering modelled by the multiple-scattering distribution functions over the long steps, and is essential for permitting tracking across sharp heterogeneities.

In these as well as other features, DPM exploits the small dynamic range (in energy and material) of radiotherapy class problems. Energies are limited to those between $\sim 100 \mathrm{keV}$ and $\sim 20 \mathrm{MeV}$, and, while the program has been benchmarked against a wide range of atomic numbers for completeness, because in most clinical applications only a few low-atomic-number materials are seen, certain cross sections and distribution functions are determined by scaling them appropriately to exactly computed data for water.

In the following sections, we present in detail the multiple-scattering model, electron transport mechanics, treatment of large energy loss processes, the photon transport algorithm, and cross-voxel transport found in DPM. Results from electron dose deposition simulations in homogeneous and inhomogeneous phantoms, as well as in a CT geometry, are then presented, followed by a section devoted to the analysis of CPU run time and of the simulation efficiency achieved.

\section{Multiple scattering}

In all condensed history MC programs, the effect of the large number of elastic interactions which occur over a given pathlength is modelled by means of a multiple scattering theory. The theory of Goudsmit and Saunderson (1940a) (GS hereafter), which is exact if the cross section is constant over the step, describes the angular deflection of electrons after travelling a given pathlength $s$ in terms of transport coefficients $g_{\ell}(\ell=0,1 \ldots \infty)$, defined by

$$
g_{\ell}=1-\int_{-1}^{1} \mathrm{~d} \omega P_{\ell}(\omega) p(\omega)
$$

Here $\omega=\cos \theta$ is the angular deviation with respect to the initial electron direction, $P_{\ell}$ is the $\ell$ th Legendre polynomial and the quantity

$$
p(\omega) \equiv \frac{\sigma(\omega)}{\int_{-1}^{1} \mathrm{~d} \omega^{\prime} \sigma\left(\omega^{\prime}\right)}
$$

represents the probability density function (PDF) associated with the single-event differential cross section (DCS) $\sigma(\omega)$. Under this formalism, the angular distribution of electrons having traversed a distance $s$ is given by

$$
F_{\mathrm{GS}}(\omega) \mathrm{d} \omega=\sum_{\ell=0}^{\infty}\left(\ell+\frac{1}{2}\right) P_{\ell}(\omega) \exp \left(-\frac{s}{\lambda} g_{\ell}\right) \mathrm{d} \omega
$$

where $\lambda$ is the elastic scattering mean free path (MFP) $1 / N \sigma$, given that $N$ is the atom density of the medium and $\sigma$ is the total microscopic cross section. An important consequence of this formalism is that the transport coefficients fully characterize the elastic scattering process. Note that this series diverges for $\omega=1$, because of the presence of uncollided particles. Numerically, this divergence appears as an instability in $F_{\mathrm{GS}}$ when values of $s / \lambda$ (the number of MFPs along $s$ ) fall below 100.

As the GS model is exact only in as much as the transport coefficients are exact, $\sigma(\omega)$ must be chosen carefully. Scattering from a screened Rutherford potential, though not rigorously 
accurate, provides a physically sound model for single elastic collisions, and leads to a PDF

$$
p_{\mathrm{R}}(\omega)=\frac{2 \eta(1+\eta)}{(1+2 \eta-\omega)^{2}}
$$

where $\eta$ is related to the screening parameter in the Rutherford potential. An advantage of using the screened potential PDF is that analytical expressions for all of the transport coefficients can be derived in terms of $\eta$. Further, $\eta$ can be arbitrarily set so as to reproduce the first transport coefficient $g_{1}$ obtained from numerical integration of more accurate (and computationally cumbersome) DCSs found elsewhere (Mayol and Salvat 1997). Typical values of $\eta$ for water in the energy range relevant for radiotherapy fall in the interval from $10^{-8}$ to $10^{-4}$. In practice, a thousand coefficients are enough to ensure the convergence of the GS series except for very small pathlengths, and the approximate small momentum method of Kawrakow and Bielajew (1998b) can be used to calculate these coefficients.

Direct use of the screened Rutherford cross section and the GS theory, however, requires an impractical amount of computer memory to store accurate numerical representations of the resulting steeply forward-peaked distribution. Following Kawrakow and Bielajew (Kawrakow and Bielajew 1998b), this difficulty can be overcome by a change to a new angular variable $u$, which is defined so that the relation

$$
\left|\frac{\mathrm{d} u}{\mathrm{~d} \omega}\right|=\frac{2 B(1+B)}{(1+2 B-\omega)^{2}}
$$

is fulfilled, where $B$ represents a free parameter that is called the 'broad screening' parameter, to associate it with the screened Rutherford shape. Moreover, if the boundary conditions

$$
u(\omega=1)=0 \quad \text { and } \quad u(\omega=-1)=1
$$

are imposed, equation (5) together with (6) fully determine $u$, and the expression for the new variable $u$ is found to be

$$
u=(1-\omega) \frac{1+B}{1+2 B-\omega} .
$$

The PDF $q(u)$ of the new variable is related with the GS distribution through

$$
F_{\mathrm{GS}}(\omega) \equiv q(u)\left|\frac{\mathrm{d} u}{\mathrm{~d} \omega}\right|=q(u) \frac{2 B(1+B)}{(1+2 B-\omega)^{2}}
$$

and so $q$ can be interpreted as a 'correction' factor that transforms a screened Rutherford PDF into the GS distribution. Equation (8) also shows that the sampling of $\omega$ values according to the $\operatorname{PDF} F_{\mathrm{GS}}(\omega)$ can be readily carried out using the rejection method, employing $q(u)$ as the rejection function.

\subsection{Optimizing $q$}

Rejection sampling for $\omega$ will be accurate and efficient and will require a manageable amount of computer memory only if $q(u)$, which depends only on $s / \lambda, \eta$ and $B$, is sufficiently smooth. The introduction of the arbitrary parameter $B$ in equation (8) provides a degree of freedom that can be exploited to manipulate the shape of $q$ to bring this about. The requirement that $q$ be as smooth as possible can be expressed mathematically as

$$
\frac{\partial}{\partial B} \int_{0}^{1} \mathrm{~d} u[1-q]^{2}=0 .
$$

Since $q(u)$ is a PDF, its integral is 1 and the former equation simplifies to

$$
\frac{\partial}{\partial B} \int_{0}^{1} \mathrm{~d} u q^{2}=0
$$




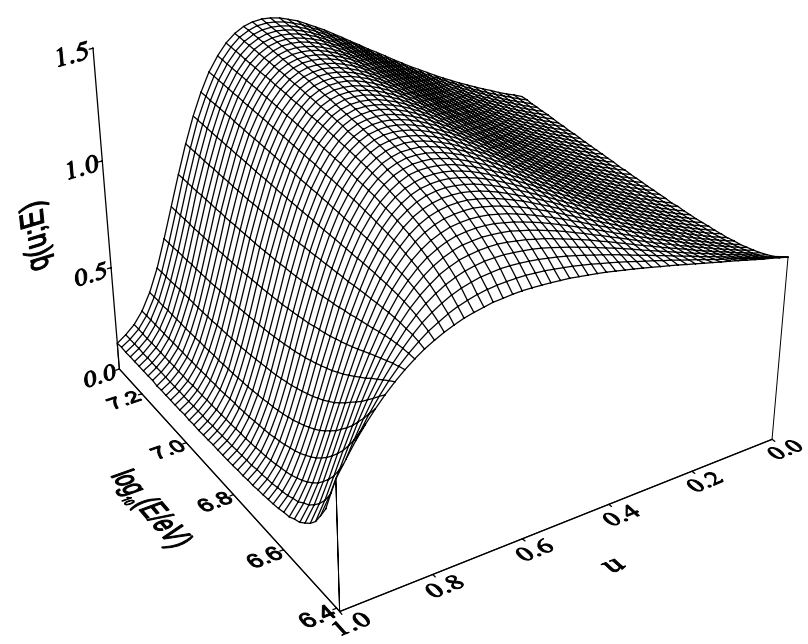

Figure 1. $q$ surface for $s=1 \mathrm{~cm}$ as a function of the angular variable $u$ and the logarithm of the kinetic energy in $\mathrm{eV}$. Note that the vertical scale is linear. The lower limit $E_{\mathrm{L}}$ of the energy interval considered is chosen to be slightly larger than energy $E_{r}$ for which the residual range is $1 \mathrm{~cm}$. At energies below $E_{r}$, the surface collapses to the plane $q=1$, and the parameter $B$ in (8) goes to $\infty$, yielding an $F_{\mathrm{GS}}$ of $1 / 2$, which implies isotropic scattering. To avoid this singularity in the interpolating routine, one must cut off at a slightly greater value, $E_{\mathrm{L}}$. This, of course, implies the need for a last-step strategy, that is, the last step is treated as a special case.

which can be solved analytically (Kawrakow and Bielajew 1998b) to obtain $B$ as a function of $s / \lambda$ and $\eta$.

A fairly good approximation for $B(s / \lambda, \eta)$ (and for the corresponding $q$ ) can be obtained in the small-angle limit, i.e. when $s / \lambda$ is not very large and the scattering is weak (equivalent to $\eta$ being small). It is noted that within this approach, which has been extensively studied by Bielajew (1994b), neither $q$ nor the ratio $B / \eta$ depend on $\eta$, making the interpolation process somewhat simpler. However, as large step sizes are required to significantly reduce computation time in DPM, the conditions necessary to apply this simplification will not be met, and a numerical solution of the exact expression in (10) is used instead.

Since $s$, as discussed in a later section, is almost always expressed as a function of the kinetic energy of the electron, $E$, and since $\lambda$ and the screening parameter $\eta$ are also functions of $E, q$ depends only on the dynamic variables $u$ and $E$. In figure 1 a plot of the surface $q(u ; E)$ is presented for $s=1 \mathrm{~cm}$, showing that the change of variable introduced in equation (7) does indeed produce a smooth $q$. Typically, less than $20 \mathrm{kB}$ (varying slightly with the selected $s(E)$ ) are needed to reproduce $q$ with a mean accuracy better than $0.1 \%$. The problem of sampling the GS distribution has thus been reduced to interpolating $q(u ; E)$.

\subsection{Multiple scattering with energy losses}

Since electrons lose energy continuously as they pass through matter and the elastic scattering cross section is a fairly strong function of the electron energy, there is dependence on energy in both $\lambda$ and $g_{\ell}$ in the exponential in (3). Lewis (1950) first accounted for this by recasting $s$ as an integral over energy loss in the continuous slowing down approximation, noting that the pathlength can be expressed in terms of energy loss as

$$
s=\int_{E-\Delta E}^{E} \frac{\mathrm{d} E}{S(E)} \equiv R(E)-R(E-\Delta E) .
$$


Here $S(E)$ is the energy-dependent energy loss per pathlength or CSDA stopping power, and $R(E)$ is called the CSDA range. The average energy loss $\Delta E$ for an electron with initial energy $E$ travelling a given distance $s$ can be determined by inverting the CSDA range, as in

$$
\Delta E=E-R^{-1}(R(E)-s) .
$$

Thus Lewis was able to introduce energy dependence into (3) by using (11) to write $s / \lambda g_{\ell}$ in the exponential as an integral over energy loss

$$
F_{\mathrm{L}}(\omega)=\sum_{\ell=0}^{\infty}\left(\ell+\frac{1}{2}\right) P_{\ell}(\omega) \exp \left(-\int_{E-\Delta E}^{E} \mathrm{~d} E \frac{G_{\ell}}{S}\right)
$$

in which

$$
G_{\ell} \equiv \frac{g_{\ell}}{\lambda}
$$

is defined as the $\ell$ th inverse transport MFP. The first inverse MFP, $G_{1}$, is often referred to as the scattering power. As $F_{\mathrm{L}}$ depends only on the dynamic variables $\omega$ and $E$, the change of variable (7) and the condition (10) can be applied as before with the GS distribution. Again, this process splits $F_{\mathrm{L}}$ into a screened Rutherford PDF and a $q(u ; E)$ surface, which now includes the effect of the energy losses within the CSDA model. Neither the memory storage nor the simulation time are significantly affected by this change. However, because the integral in (13) must be evaluated numerically for each $\ell$, the computation time required to generate the table from which $q$ will be interpolated during the execution of DPM does increase considerably. Fortunately, this needs to be done just once for a given material. It is worth noting that, unlike other schemes (Kawrakow and Bielajew 1998a), the method presented here is rigorously exact within the CSDA model, and the only approximation introduced involves the assumption of a screened Rutherford potential.

The importance of including energy losses in the multiple-scattering theory for large pathlengths is apparent from figure 2, which shows the difference in depth dose profiles for a $10 \mathrm{MeV}$ electron beam in water when the more accurate Lewis approach is used instead of the GS scheme.

\subsection{Electron transport mechanics}

Since large pathlengths must be used to attain appreciable speed up of MC electron transport computations, the mechanism used to generate final phase space variables after a transport step plays a critical role in determining the accuracy of the model. As noted earlier, the efficacy of a given transport model can be evaluated by its faithfulness in reproducing the spatial and angular moments of the phase variables and the spatial and angular distributions, at the end of a given step. A comparison of transport mechanics methods has been performed by Larsen (1992) and by Kawrakow and Bielajew (1998a). They concluded that when the energy loss along a step is disregarded, PENELOPE's random hinge model (Fernández-Varea et al 1993) provides an excellent compromise between speed and accuracy, and is therefore well suited for a fast MC code. The random hinge transport method is described as follows. The pathlength $s$ is split in two substeps of lengths

$$
s_{\mathrm{A}}=\xi s \quad \text { and } \quad s_{\mathrm{B}}=s-s_{\mathrm{A}}
$$

respectively, where $\xi$ is a random number between 0 and 1. A first substep $s_{\mathrm{A}}$ is taken in the initial electron direction, after which the particle is deflected according to any multiplescattering law which provides polar and azimuthal deflection angles $\Theta$ and $\Phi$ determined over the entire step $s$. A second substep is then taken over the remaining distance $s_{\mathrm{B}}$ in the new direction. For a particle directed along the $z$-axis starting at location $\vec{x}=0$, provided that 


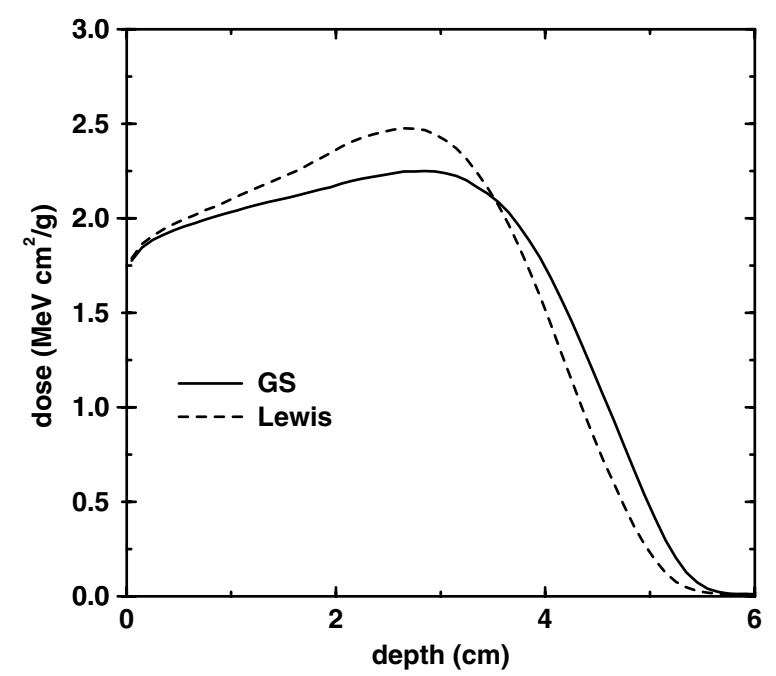

Figure 2. Depth dose profiles of a $10 \mathrm{MeV}$ electron beam in water calculated showing the effect of substituting a model based on the GS theory (full curve) by another based on the Lewis' theory (broken curve). The pathlength was set to $1 \mathrm{~cm}$.

the scattering law is correct and disregarding energy losses along the step, it can be shown that this method yields average values of the normalized penetration depth $z / s$ and lateral displacement $\left(x^{2}+y^{2}\right) / s^{2}$, which are correct to $\mathrm{O}(s)$. Other moments preserved to this order accuracy include $\left(z v_{z}\right) / s$ (for $\left.|v|=1\right),\left(x v_{x}+y v_{y}\right) / s, z^{2} / s^{2}$ and $\left(x^{2}+y^{2}\right) z / s^{3}$.

The inclusion of energy losses along $s$ reduces the accuracy of the random hinge model. Indeed, Larsen's analysis of the spatial moments is valid only when the scattering power, $G_{1}$, and other inverse transport MFPs do not depend on $s$, or equivalently, on the energy $E$. For long steps $s$, these conditions will not be met and a modified formulation of random hinge mechanics must be employed. We begin by noting that exact Lewis moments (indicated by $\left.\langle\cdot\rangle_{\mathrm{L}}\right)$ under the CSDA energy-loss model are given by

$$
\frac{\langle z\rangle_{\mathrm{L}}}{s}=\frac{1}{s} \int_{0}^{s} \mathrm{~d} s^{\prime} \exp \left[-K_{1}\left(s^{\prime}\right)\right] \simeq 1-\frac{1}{s} \int_{0}^{s} \mathrm{~d} s^{\prime} K_{1}\left(s^{\prime}\right) \simeq 1-\frac{1}{2} s G_{1}\left(\frac{s}{3}\right)
$$

for the penetration depth and

$$
\begin{aligned}
\frac{\left\langle x^{2}+y^{2}\right\rangle_{\mathrm{L}}}{s^{2}}= & \frac{4}{3 s^{2}} \int_{0}^{s} \mathrm{~d} s^{\prime} \exp \left[-K_{1}\left(s^{\prime}\right)\right] \int_{0}^{s^{\prime}} \mathrm{d} s^{\prime \prime} \exp \left[K_{1}\left(s^{\prime \prime}\right)\right]\left(1-\exp \left[-K_{2}\left(s^{\prime \prime}\right)\right]\right) \\
& \simeq \frac{4}{3 s^{2}} \int_{0}^{s} \mathrm{~d} s^{\prime} \int_{0}^{s^{\prime}} \mathrm{d} s^{\prime \prime} K_{2}\left(s^{\prime \prime}\right) \simeq \frac{2}{9} s G_{2}\left(\frac{s}{4}\right)
\end{aligned}
$$

for the lateral displacement. $K_{\ell}$ is defined as

$$
K_{\ell}(s) \equiv \int_{0}^{s} \mathrm{~d} s^{\prime} G_{\ell}\left(s^{\prime}\right) \simeq s G_{\ell}\left(\frac{s}{2}\right)
$$

with $K_{1}$ called the 'scattering strength'. These relations use a first-order Taylor expansion for $G_{\ell}(s)$, and neglect terms containing products of two or more $K_{\ell}$ s. This gives an upper bound for the Lewis lateral displacement and an acceptable approximation for values of $s$ not extremely large and energies not too low. If energy loss is taken into account in sampling $\cos \theta$ 
and PENELOPE's mechanics (equation (15)) is used otherwise, the random hinge moments can be computed to be (Fernández-Varea et al 1993)

$$
\frac{\langle z\rangle_{\mathrm{P}}}{s}=\frac{1+\langle\cos \theta\rangle}{2}=\frac{1+\exp \left[-K_{1}(s)\right]}{2} \simeq 1-\frac{1}{2} s G_{1}\left(\frac{s}{2}\right)
$$

and

$$
\frac{\left\langle x^{2}+y^{2}\right\rangle_{\mathrm{P}}}{s^{2}}=\frac{1}{3}\left(1-\left\langle\cos ^{2} \theta\right\rangle\right)=\frac{2}{9}\left(1-\exp \left[-K_{2}(s)\right]\right) \simeq \frac{2}{9} s G_{2}\left(\frac{s}{2}\right) .
$$

We therefore see that the effect of ignoring energy loss in the transport mechanics is equivalent to evaluating the $G_{\ell}(s)$ s at the step mid-point rather than the correct distances of $s / 3$ or $s / 4$. As $G_{\ell}(s)$ increases as $s$ increases (and $E$ decreases), $\langle z\rangle_{\mathrm{P}}$ slightly underestimates the true value $\langle z\rangle_{\mathrm{L}}$, and $\left\langle x^{2}+y^{2}\right\rangle_{\mathrm{P}}$ substantially overestimates $\left\langle x^{2}+y^{2}\right\rangle_{\mathrm{L}}$. In physical terms, the PENELOPE model overestimates the scattering for very large pathlengths. Computations of these moments for $10 \mathrm{MeV}$ electrons in water with a pathlength of $1 \mathrm{~cm}$ show that the PENELOPE model is in error by less than $0.1 \%$ for the penetration $\langle z\rangle$, but gives an excess lateral displacement of about $3 \%$. These discrepancies increase with decreasing energy, with the lateral displacement error rising to approximately $10 \%$ at $4 \mathrm{MeV}$. Moreover, this deviation is systematic and compounds as the total pathlength travelled by the electron increases.

The above analysis suggests a modification of the random hinge model which preserves the Lewis moments by sampling uniformly in scattering strength $K_{1}^{(\mathrm{A})}$ rather than in distance $s$, i.e.

$$
K_{1}^{(\mathrm{A})}=\xi K_{1}(s)
$$

An electron is then transported until it 'accumulates' a scattering strength equal to $K_{1}^{(\mathrm{A})}$, where a deflection is imposed. The electron is then moved the pathlength required to exhaust the scattering strength $K_{1}(s)-K_{1}^{(A)}$. It can be shown that this non-uniform PDF for the first substep distance $s_{\mathrm{A}}$ yields correct values for the average penetration depth, lateral displacement and other spatial moments to first order in $s G_{1}$ and $s G_{2}$ when a linear approximation is adopted for $G_{\ell}(s)$. Perhaps more importantly, in addition to correcting for the scattering overestimation, this new transport mechanism also provides a basis for simulating scattering across material or density boundaries. Time saved in multi-voxel transport offsets by far the additional bookkeeping required in calculating the $K_{1}$ accumulated over the steps. The details of the transport through voxels is presented in a subsequent section.

\section{Discrete electron energy loss interactions}

DPM employs what Berger (1963) has categorized as a class II mixed simulation scheme for energy losses. Hard interactions, i.e. those yielding energy loss above given cut-offs, are simulated discretely using an analogue (event-by-event) model. Soft events, which are much more frequent but result in energy transfer below the cut-offs, are modelled as contributing to a continuous deposition of energy throughout the transport step, and are accounted for in the CSDA approximation through the use of a restricted stopping power, as described later. In addition to resulting in energy loss to the primary electron, hard ionization events generate secondary electrons and hard bremsstrahlung collisions generate secondary photons. The energy loss of the primary and the phase state of the secondary particles is generated by sampling from the appropriate PDFs describing the processes. 


\subsection{Hard inelastic collisions}

DPM uses the Møller DCS, $\sigma_{\mathrm{M}}$, to treat inelastic collisions of electrons with atomic electrons. The Møller cross section, which was derived for collisions with free electrons at rest, is given by

$\sigma_{\mathrm{M}}(k)=\frac{2 \pi e^{4}}{m v^{2}} \frac{Z}{E k^{2}}\left[1+\left(\frac{k}{1-k}\right)^{2}-\frac{k}{1-k}+\left(\frac{\gamma-1}{\gamma}\right)^{2}\left(k^{2}+\frac{k}{1-k}\right)\right]$

where $e$ is the charge of the electron, $m$ its rest mass, $v$ its speed, $\gamma$ is the ratio of its total energy $E+m c^{2}$ to its rest energy, $Z$ is the number of electrons in the target molecule and $k=W / E$ is the fraction of kinetic energy lost. Note that in the Møller formalism, the maximum allowed value of $k$ is $1 / 2$ due to the indistinguishability of the projectile and target electrons.

The inverse MFP $\lambda_{\mathrm{M}}^{-1}$ for hard inelastic events (those above the cut-off $W_{\mathrm{M}}$ ) in homogeneous media is easily derived by integrating (22)

$\lambda_{\mathrm{M}}^{-1}=\frac{2 \pi \mathrm{e}^{4}}{m v^{2}} \frac{Z N}{E}\left\{\frac{1-2 k_{\mathrm{M}}}{k_{\mathrm{M}}\left(1-k_{\mathrm{M}}\right)}+\left(\frac{\gamma-1}{\gamma}\right)^{2}\left(\frac{1}{2}-k_{\mathrm{M}}\right)+\left[\left(\frac{\gamma-1}{\gamma}\right)^{2}-1\right] \ln \frac{1-k_{\mathrm{M}}}{k_{\mathrm{M}}}\right\}$

where $N$ is the number of molecules per unit volume and

$$
k_{\mathrm{M}} \equiv \frac{W_{\mathrm{M}}}{E} \text {. }
$$

In the limit that $m c^{2} \ll E$ and $k_{\mathrm{M}} \ll 1$, equation (23) can be approximated as

$$
\lambda_{\mathrm{M}} \simeq \frac{A}{Z \rho} \frac{m c^{2} W_{\mathrm{M}}}{N_{A} 2 \pi e^{4}}
$$

where $A$ is the atomic weight of the species and $N_{A}$ is Avogadro's number. By default, DPM sets $W_{\mathrm{M}}=200 \mathrm{keV}$, as knock-on electrons with less than that energy have ranges much smaller than the minimum $1 \mathrm{~mm}$ voxel size. For water, (23) yields a value of $\lambda_{\mathrm{M}}$ roughly equal to $2 \mathrm{~cm}$ and practically independent of $E$, as shown by equation (25). The simple $Z \rho / A$ dependence on medium composition implicit in (23) will be exploited later in transport across voxel boundaries.

When a Møller interaction takes place, the fraction $k$ of energy lost is sampled from the normalized PDF based on (22) by combining the rejection and composition methods (Salvat and Fernández-Varea 1992), and a knock-on electron is generated and its energy, direction and position stored for later transport. Since energy losses are usually much larger than the binding energies, the approximation that target electrons are initially free and at rest is appropriate. A knock-on electron will then have a kinetic energy equal to $W$ and a direction of movement determined by the conservation of momentum. Naming $\theta_{2}$ as the angle formed between this direction and the velocity of the incoming electron, it is found that

$$
\cos \theta_{2}=\sqrt{\frac{W\left(E+2 m c^{2}\right)}{E\left(W+2 m c^{2}\right)}} .
$$

\subsection{Hard bremsstrahlung interactions}

The bremsstrahlung DCS for an electron impinging on a neutral atom with $Z$ electrons to produce a photon with energy $W=k E$ can be written as

$$
\sigma_{\mathrm{B}}(k)=\frac{Z^{2}}{\beta^{2} k} f(k)
$$


where $\beta$ is the electron velocity in units of the speed of light. Except for very high values of $Z$ and low values of $E$, the leading term in (27) removes almost all the dependence of $\sigma_{\mathrm{B}}(k)$ on $E$ and $Z$, and the correction $f$ is a smooth function of $k$. Seltzer and Berger (1985) have given a tabulation of $f(k)$ in terms of $Z$ and $E$ for selected $Z$ values. For materials and energies typically seen in radiotherapy problems, the data contained in these tables can be roughly approximated by means of a linear function

$$
f(k)=a(1-b k)
$$

with $a$ and $b$ being material- and energy-independent constants selected by performing a fit to the tabulated data. Inaccuracies in this approximation have little effect for most problems of interest, as the parameters $a$ and $b$ are weak functions of $E$ and $Z$ in the radiotherapy regime.

For compounds or mixtures, DPM relies on the additivity rule, replacing $Z^{2}$ in equation (27) with

$$
Z_{\mathrm{eq}}^{2} \equiv \sum_{i} q_{i} Z_{i}^{2}
$$

where $q_{i}$ and $Z_{i}$ represent the stoichiometric index and the atomic number of the $i$ th atom respectively. (For the sake of simplicity, the symbol $Z^{2}$, will be used throughout in place of $Z_{\mathrm{eq}}^{2}$.)

For a given cut-off energy for bremsstrahlung production $W_{\mathrm{B}}$, the inverse MFP resulting from this approximate DCS is

$$
\lambda_{\mathrm{B}}^{-1}=\frac{Z^{2} n_{m} a}{\beta^{2}}\left(\ln \frac{1}{k_{\mathrm{B}}}-b\left(1-k_{\mathrm{B}}\right)\right)
$$

where $k_{\mathrm{B}}=W_{\mathrm{B}} / E$. In the limit $m c^{2} \ll E$ and $k_{\mathrm{B}} \ll 1$, equation (30) can be approximated as

$$
\lambda_{\mathrm{B}} \simeq \frac{A}{Z^{2} \rho N_{A} a}\left(\ln \frac{E}{W_{\mathrm{B}}}-b\right)^{-1}
$$

which shows that $\lambda_{\mathrm{B}}$ has a mild variation with $E$ at high energies. This fact, along with the linear scaling of $\lambda_{\mathrm{B}}$ with $Z^{2} \rho / A$ will be used to facilitate cross-voxel transport.

The analogue simulation of hard bremsstrahlung events, despite their infrequent occurrence, is necessary to accurately reproduce the fluctuations of the kinetic energy of impinging electrons. As the Møller DCS depends on the energy loss roughly as $k^{-2}$ and the bremsstrahlung DCS as $k^{-1}$ (equations (22) and (27) respectively), large energy losses are more likely to happen when the latter type of interaction occurs. As a result, a non-negligible fraction of incident electron energy straggling is caused by bremsstrahlung.

The random sampling of the PDF corresponding to the normalized $\sigma_{\mathrm{B}}(k)$ can be performed using $f(k)$ in equation (27) as a rejection function. The angular deflection of the incoming electron is small and can be neglected and the scattering angle of the secondary photon is set equal to its mean value, approximately given by (Heitler 1954)

$$
\langle\theta\rangle \simeq \frac{m c^{2}}{E+m c^{2}}
$$

which is the approximation adopted in the original version of EGS.

\section{Photon interactions}

Photon transport is described following a conventional analogue MC treatment until the energy falls below some user-defined absorption energy. Three processes, photoelectric absorption, Compton scattering and pair production, are considered. The inverse MFPs for 
these interactions are taken from those generated by the PENELOPE preprocessing program MATERIAL. In radiotherapy class problems, Compton scattering is the only significant dose delivery mechanism, and so approximations have been adopted for treating photoelectric and pair production interactions.

Photoelectric absorption, which is relevant only at very low energies and for high atomic numbers, is simulated by assuming that all the energy is locally deposited. DPM does not generate secondary electrons or relaxation radiation, so it is therefore convenient to set the electron and photon absorption energies above the highest absorption edge of the highest $Z$ material in the problem. In most applications, DPM uses absorption thresholds of $50 \mathrm{keV}$ for photons and $200 \mathrm{keV}$ for electrons.

Pair production is important only at the high end of the energy range relevant to radiotherapy, and only for high atomic numbers, and so some very rough approximations are made. DPM assumes that for the first emerging particle, all kinetic energies are equally probable, and generates two electrons travelling in the same direction as the incident photon. Both particles are tracked as electrons, and one is randomly selected upon stopping to emit two annihilation photons travelling in randomly selected opposite directions. This approach disregards the differences in the cross sections and stopping powers between the created electron and positron (and the small possibility of in-flight annihilation of the positron), approximations which are justified by the relatively small impact of this effect in practical problems.

Compton interactions are assumed to involve free electrons at rest, and therefore binding effects (accounted for by means of the incoherent scattering function) and the Doppler broadening (Ribberfors 1975) of the energy of the scattered photon are ignored. These approximations are in general excellent for energies above $1 \mathrm{MeV}$, and are even more applicable to radiotherapy problems, where the only sources of low-energy photons are either contamination in the accelerator head and relatively rare hard bremsstrahlung interactions. DPM determines the energy of the scattered photon by sampling the Klein-Nishina DCS using the recipe contained in EGS4 (Nelson et al 1985). The recoil electron, which has direction and energy determined by the energy and momentum conservation laws, is stored in the secondary stack and simulated afterwards.

Photon histories terminate when the energy falls below a user-defined absorption energy or when they reach the geometry limits.

\section{Transport across inhomogeneous voxel boundaries}

As noted previously, a Monte Carlo electron transport algorithm sufficiently fast for clinical radiotherapy treatment planning will require the use of transport steps significantly greater than patient geometry voxel dimensions. This is problematic, as patient geometry (composition and density), which is typically inferred from $\mathrm{CT}$ data $\dagger$, varies across almost every voxel boundary. Conventional MC programs are not capable of single-step transport over boundaries between differing media because the cross sections used in their multiple-scattering laws are medium dependent. Because DPM uses the medium-invariant $q(u)$ function to describe multiple scattering, however, transport across inhomogeneities is possible, as described below.

$\dagger$ Following the method of Knöös et al (1986), the program CTCREATE (Ma et al 1995) has been developed for the OMEGA BEAM (Rogers et al 1995) project, and is publicly available. 


\subsection{Electrons}

The atomic number and density dependences of the physical models adopted by DPM have been examined in detail in the preceding sections. Here we describe how the particular forms of these quantities can be exploited to permit rapid simulation of electron transport in voxelized geometries.

In typical mixed class II electron transport MC models, electrons are started in an initial direction with an initial energy and transported in a series of steps until they exit the problem geometry or their energies fall below a user defined absorption cut-off. A transport step involves linear translation of the particle along its direction vector until a boundary is crossed, a hard collision takes place, or a multiple elastic scattering event is imposed. The details of how DPM determines when various events occur is presented here. Note that the computations described below of the distances travelled prior to the simulation of the different events are done in parallel as the electrons traverse the voxels.

- The distance to a Møller collision is sampled according to

$$
t_{\mathrm{M}}=-\lambda_{\mathrm{M}} \ln \xi
$$

where $\lambda_{M}$ is the Møller MFP, equation (23), for some reference material (which will be assumed to be, without loss of generality, pure water) and $\xi$ represents a random number uniformly distributed in $(0,1)$. A look-up table with values of $\lambda_{M}$ on a grid of energies dense enough to allow accurate numerical interpolation is calculated beforehand and read from an input file during the initialization of DPM.

When an electron travels a distance $t$ inside a voxel, $t_{\mathrm{M}}$ is decreased an amount $\Delta t_{\mathrm{M}}$ given by

$$
\Delta t_{\mathrm{M}}=t \frac{(Z \rho / A)_{\mathrm{vox}}}{(Z \rho / A)_{\mathrm{water}}} .
$$

This is continued at each voxel until $t_{\mathrm{M}}$ drops to zero. A Møller interaction is then simulated, in which the energy lost by the incident electron is sampled from the Møller DCS of (22), and a knock-on electron is generated and placed in the secondary stack. Note that for a homogeneous medium made of water, the scattering event occurs when the distance $t$ accumulated over voxels equals $t_{\mathrm{M}}$.

- The distance to a bremsstrahlung collision is sampled according to

$$
t_{\mathrm{B}}=-\lambda_{\mathrm{B}} \ln \xi
$$

where $\lambda_{B}$ is the bremsstrahlung MFP, equation (30), for the reference material, i.e. for water. Again, an interpolation table is generated beforehand for $\lambda_{\mathrm{B}}$ and read from an input file during the initialization of DPM.

When an electron travels a distance $t$ inside a voxel, $t_{\mathrm{B}}$ is decremented by an amount equal to

$$
\Delta t_{\mathrm{B}}=t \frac{\left(Z^{2} \rho / A\right)_{\mathrm{vox}}}{\left(Z^{2} \rho / A\right)_{\text {water }}}
$$

and this process is repeated until $t_{\mathrm{B}}$ drops to zero. At that point a radiative event is simulated, in which a photon is generated with an energy sampled from the bremsstrahlung DCS of equation (27) and placed in the secondary stack. For a homogeneous medium made of water, the interaction takes place when the total distance $s$ travelled across voxels equals $t_{\mathrm{B}}$. 
- The total scattering strength $K_{1}$, given by equation (18) for $\ell=1$, is obtained for water at the electron energy at the midpoint of the step. Values of $K_{1}$ as a function of energy and a preset pathlength $s$ are precalculated and read by DPM during its initialization. The scattering strength prior to simulation of a multiple-scattering event is then sampled as

$$
t_{\mathrm{S}}=K_{1}^{(\mathrm{A})} \equiv \xi K_{1}
$$

in accordance with the corrected version of the PENELOPE transport mechanics described earlier (see equation (21)). It must be stressed that the units of $t_{\mathrm{S}}$ are not those of distance, but of scattering strength.

As each step $t$ is taken inside a voxel, the scattering strength prior to multiple scattering, $t_{\mathrm{S}}$, is decreased by an amount equal to

$$
\Delta t_{\mathrm{S}}=\int_{0}^{t} \mathrm{~d} t^{\prime} G_{1}^{(\mathrm{vox})}\left(t^{\prime}\right) \simeq \frac{t}{2}\left[G_{1}^{(\mathrm{vox})}\left(t^{\prime}=0\right)+G_{1}^{(\operatorname{vox})}\left(t^{\prime}=t\right)\right] .
$$

Once $t_{\mathrm{S}}$ is exhausted, the angular deviation is sampled from the Lewis PDF, as described in previous sections, using the $q(u ; E)$ surface corresponding also to water. After rotating through the scattering angle to determine the new electron direction, linear transport is resumed until a new quantity of scattering strength, given by

$$
t_{\mathrm{S}}=K_{1}^{(\mathrm{B})} \equiv K_{1}-K_{1}^{(\mathrm{A})}
$$

is spent. After the distance corresponding to $t_{\mathrm{S}}$ (as determined by summing the $\Delta t_{\mathrm{S}}$ incurred while stepping through each voxel) is traversed, the process is repeated, with a new total scattering strength $K_{1}$ determined by table look-up and a new $t_{\mathrm{S}}$ sampled according to (37). This procedure ensures that the actual pathlength is such that it produces the same mean angular deviation as over a predefined reference pathlength for water.

- Apart from discrete events, the continuous energy loss of the electrons is computed at each step. This is given by

$$
\Delta E=\int_{0}^{t} \mathrm{~d} t^{\prime} S_{\mathrm{r}}^{(\operatorname{vox})}\left(t^{\prime}\right)
$$

where $t$ is the distance traversed in a given voxel prior to a hard collision or exiting the voxel and $S_{\mathrm{r}}^{(\mathrm{vox})}$ is the stopping power in the medium, 'restricted' to energy transfers below the Møller and bremsstrahlung production thresholds for the problem. For large $t, S_{\mathrm{r}}^{\text {(vox) }}$ can vary over the step, and so the integral is approximated by first estimating the energy loss over $t$ assuming that $S_{\mathrm{r}}^{(\mathrm{vox})}$ is constant, and then averaging the stopping power over the step, as in

$$
\Delta E=t \frac{\left.S_{\mathrm{r}}^{(\mathrm{vox})}\right|_{E_{0}}+\left.S_{\mathrm{r}}^{(\mathrm{vox})}\right|_{E_{0}-t S_{\mathrm{r}}^{(\mathrm{vox})}}}{2} .
$$

Here $E_{0}$ is the electron kinetic energy at the beginning of the step and $E_{0}-t S_{\mathrm{r}}^{(\mathrm{vox})}$ is what the energy would be if the stopping power were constant. Values of $S_{\mathrm{r}}^{(\mathrm{vox})}$ are precalculated for a dense grid of energies and read by DPM during its initialization.

It should be noted that, since hard inelastic MFPs depend on the energy, the exact sampling of the distance $s$ to the next interaction of type ' $\mathrm{i}$ ' is given by

$$
\xi=\int_{0}^{t} \mathrm{~d} t^{\prime} \lambda_{\mathrm{i}}^{-1}\left(t^{\prime}\right) \exp \left[-\int_{0}^{t^{\prime}} \mathrm{d} t^{\prime \prime} \lambda_{i}^{-1}\left(t^{\prime \prime}\right)\right] .
$$

Equations (33) and (35) assume that $\lambda(t) \simeq$ constant across a voxel, which is computationally cheap, quite good for Møller collisions, and reasonably good for bremsstrahlung interactions. However, because of the possibility of large energy loss occurring in a hard collision, the energy 
dependence of $\lambda(t)$ cannot be ignored when determining distances to additional interactions, and so $t_{\mathrm{M}}$ and $t_{\mathrm{B}}$ are recomputed individually whenever either type of hard collision takes place.

The electron history terminates when it leaves the CT geometry or when its energy falls below a user-defined absorption energy, $E_{\text {abs }}$, set by default to $200 \mathrm{keV}$ that is the approximate energy at which the electron CSDA range equals $1 \mathrm{~mm}$, a typical voxel size.

One important advantage of this algorithm over conventional MC electron transport schemes is that the scaling of the cross sections precludes expensive table look-ups when each new voxel is encountered. More significantly, the number of multiple-scattering events is dramatically reduced. In a conventional scheme, electrons are deflected not only when a multiple scatter step is traversed, but also at every boundary crossing and prior to the simulation of every hard inelastic collision. Thus DPM eliminates the majority of the computationally expensive samples from the MS distributions and rotations through the scattering angles.

\subsection{Photons}

Since photons undergo a limited number of interactions before they are locally absorbed, their transport is almost always treated in an analogue manner. This requires that the distance to collision be recomputed at every medium boundary, as, unlike the case of electrons, there are no simple scaling laws which can be applied. Since voxelized geometries can present frequent changes of material in short distances (relative to photon MFPs), this imposes a significant speed penalty.

To overcome this difficulty, DPM uses the $\delta$-scattering method of Woodcock et al (1965), which avoids calculating intersections with the interfaces of all the visited voxels by exploiting the fact that the distribution of collision distances $t$ contains the product of the probability of not colliding prior to $t$ and the collision density. The method is implemented by first determining the energy-dependent minimum total MFP $\lambda_{\gamma}^{(\min )}(E)$ in the entire geometry. A distance to the next interaction $t$ is then sampled using $\lambda_{\gamma}^{(\min )}(E)$, and the photon is transported through $t$, ignoring all boundary crossings. Next, the material of the current voxel is determined, which is simple and efficient for voxelized geometries. An interaction is simulated at $t$ only with probability $\mathcal{P}$ equal to

$$
\mathcal{P}=\frac{\lambda_{\gamma}^{(\min )}}{\lambda_{\gamma}^{(\operatorname{vox})}}
$$

where $\lambda_{\gamma}^{(\text {vox) }}$ represents the total MFP in the current voxel. If an interaction does not occur, the transport is continued. The quantity $1-\mathcal{P}$, which is easily determined for every voxel, can be considered to be the probability of a 'fictitious' event occurring, in which no phase space change takes place. If an interaction does occur, its type is sampled according to the corresponding probabilities $\mathcal{P}_{\mathrm{i}}$ (with 'i' representing Compton collision, photoelectric absorption or pair production), which are

$$
\mathcal{P}_{\mathrm{i}}=\frac{\lambda_{\gamma}^{(\mathrm{vox})}}{\lambda_{\mathrm{i}}^{(\mathrm{vox})}} .
$$

$\lambda_{i}^{(\text {vox) }}$ represents the MFP of the interaction ' $i$ ' in the current voxel. The Woodcock method will be efficient if there is only slight variation in $\lambda$ throughout the geometry, and so time expended in stopping to analyse collisions which are then determined to be fictitious is less than the time saved by not stopping to recompute $\lambda$ at each voxel boundary. 


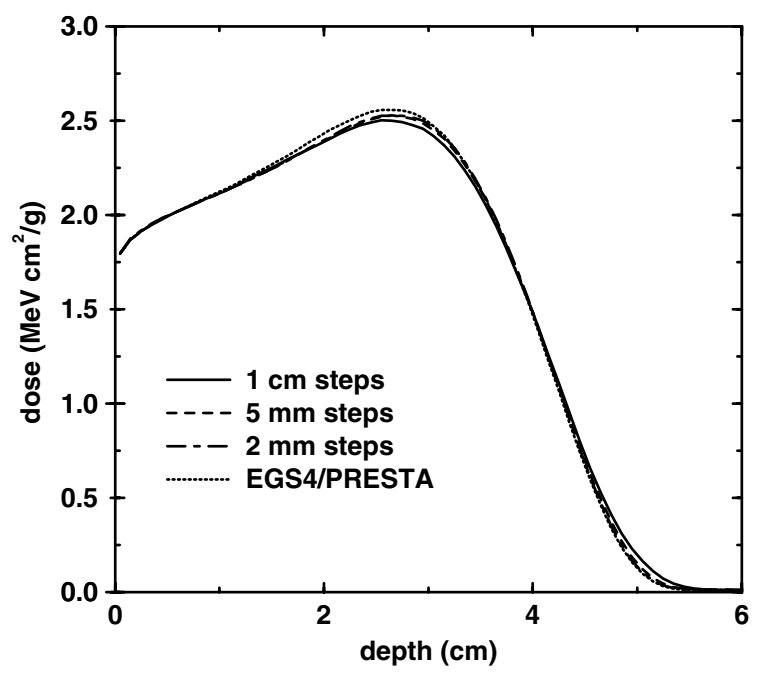

Figure 3. Depth dose produced by a $10 \mathrm{MeV}$ electron pencil beam impinging normally on a semi-infinite water phantom using various reference step sizes.

\section{Results}

Results from simulations performed using DPM are compared here with results generated by EGS4 and PENELOPE. EGS4 has been extensively benchmarked against experimental data (Rogers and Bielajew 1989b, 1990) for radiotherapy problems, and is widely accepted as a standard. PENELOPE has likewise shown excellent agreement in a variety of comparisons with experimental and other Monte Carlo results (Baró et al 1995, Sempau et al 1997). Additionally, as DPM draws much of its physics data from PENELOPE's comprehensive and easily manipulated database, any discrepancies between DPM and PENELOPE should reflect differences in algorithms rather than differences in the underlying data or physical constants and models.

In order to fully exercise the approximations in DPM, a set of problems involving both homogeneous and multi-layered geometries and a wide range of materials (including several not typically seen in radiotherapy problems) has been simulated with all three codes.

\subsection{Step size selection}

The use of the condensed history method introduces an inherent error in Monte Carlo simulations, as elegantly characterized by Larsen (1992), who showed that it vanishes as the pathlength $s$ tends to zero. But as efficient computation depends on taking long steps, ascertaining the longest multiple scattering step which preserves the accuracy of the simulation is of critical importance, and so is addressed by all conventional MC electron transport programs. Common treatments allow particles to advance until either some predetermined fraction of their initial kinetic energy is lost (e.g. PENELOPE when C2 is active, EGS4 with the ESTEPE option, ETRAN, ITS, MCNP) or until some fixed pathlength has been travelled (e.g. PENELOPE when the option HFPMAX is active, EGS4 with the SMAX option). Another frequently used technique (e.g. PENELOPE when C1 is active, EGS4 by default) limits $s$ by fixing the mean angular deviation and calculating the step size accordingly. 


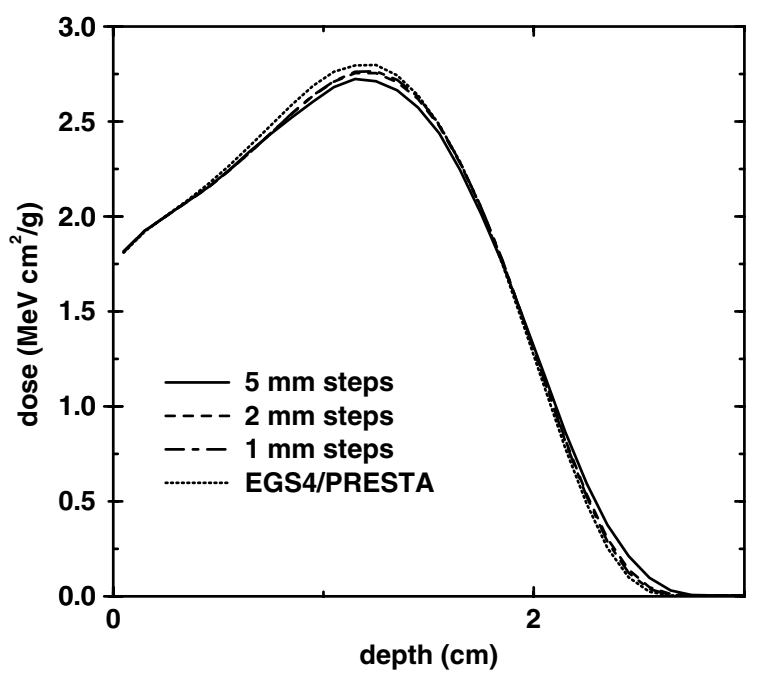

Figure 4. Depth dose produced by a $5 \mathrm{MeV}$ electron pencil beam impinging normally on a semiinfinite water phantom using various reference step sizes.

In DPM, the step size issue arises even though $q(u)$ has been shown to be a function of $u$ and $E$ rather than $s$. The energy dependence of $q$ is driven by the energy dependence of $s / \lambda(E)$, and since $\lambda(E)$ is fixed by the reference material, in order to use precomputed tabulated values of $q(u)$ in sampling for $\omega, s$ must be set in advance. The maximum value of $s$ which preserves accuracy in a simulation can be determined by comparing simulation results for increasingly smaller step sizes. In figure 3, we show depth dose curves computed by DPM for one million $10 \mathrm{MeV}$ electrons incident on a homogeneous phantom of $128^{3}$ $1 \mathrm{~mm}$ voxels, using $q(u)$ and $K_{1}$ calculated with step sizes ranging from $1 \mathrm{~cm}$ to $1 \mathrm{~mm}$. There is little appreciable difference in the results for steps shorter than $5 \mathrm{~mm}$. Note that because of the computation of energy loss in each voxel and the modelling of hard inelastic collisions, the computing time for a simulation is not directly proportional to the number of steps. The CPU usage for the simulations shown in this figure increase by only a factor of two while the step size decreased tenfold. At lower energies, there is more scatter for the same distance $s$, and the use of long steps maximizes the underlying condensed history error, as is seen in figure 4 . In the first case $(s=5 \mathrm{~mm})$, the fall of the DPM curve occurs just after completing the second step, reflecting the failure of the last one or two steps in reproducing the remaining part of the depth dose. This behaviour disappears when the pathlength is reduced to 1 or $2 \mathrm{~mm}$. From these two sets of results, we determined that roughly $8-10$ scattering events per history are necessary to reproduce depth dose profiles accurately, and that no further accuracy is attained by moving to steps of 1/20th of the range. By contrast, EGS4 requires the simulation of several multiple scattering events in every voxel traversed by the electron.

\subsection{Homogeneous phantoms}

Figures 5 to 9 show deposited depth dose curves for electron beams in semi-infinite phantoms made of different materials of interest in radiotherapy. The differences between EGS4, DPM and PENELOPE are equal to or less than $1.25 \%$ of the dose maximum in all cases and the statistical uncertainty of the curves presented are of the order of $0.2 \%$ of the dose maximum, 


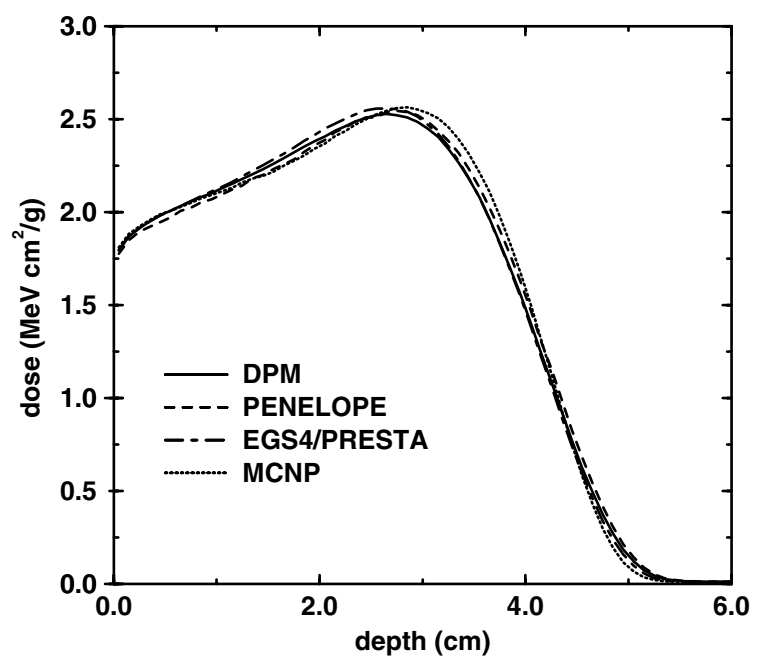

Figure 5. Depth dose produced by a $10 \mathrm{MeV}$ electron pencil beam impinging normally on a semi-infinite water phantom.

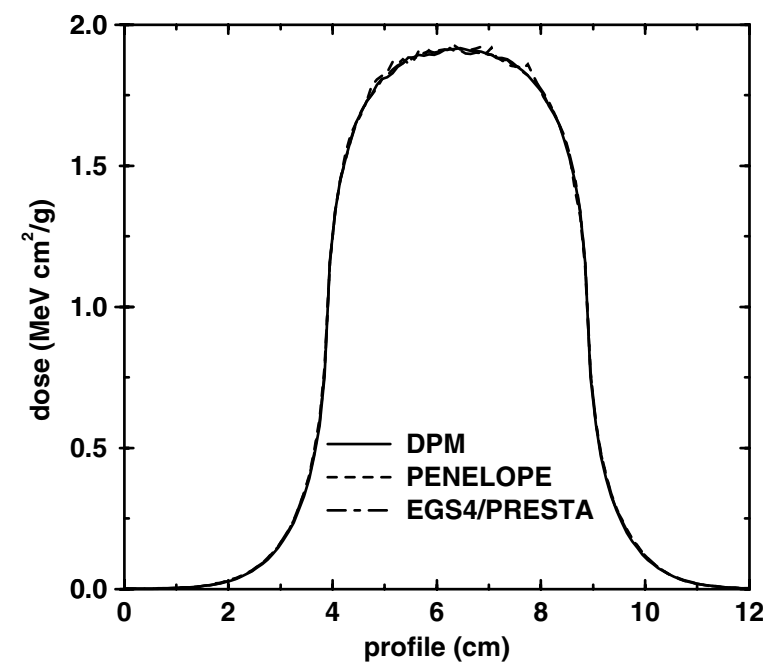

Figure 6. Dose integrated over planes perpendicular to the $y$-axis (that goes parallel to the water surface) produced by a $10 \mathrm{MeV}$ electron beam impinging normally on a semi-infinite water phantom in a $5 \times 5 \mathrm{~cm}^{2}$ field.

and step sizes of $5 \mathrm{~mm}$ are used. Energy cut-offs of $200 \mathrm{keV}$ for electrons and $50 \mathrm{keV}$ for photons were used for all cases. In figure 5, the depth dose curve computed by MCNP is also included. It is interesting to note that the results from DPM generally lie inside the envelope of the results from the other programs.

\subsection{Effects of hard collision physics approximations in DPM}

The next set of problems was specifically designed to test the limits of the approximate scaling of the hard collision cross sections used in DPM. Recall that DPM samples the distance to a 


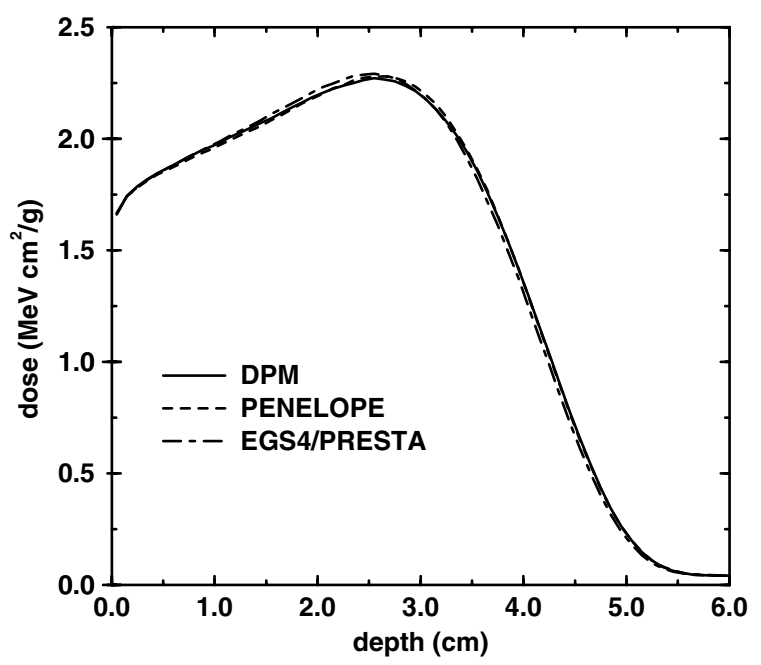

Figure 7. Depth dose for an $18 \mathrm{MeV}$ normal pencil beam in a semi-infinite bone phantom.

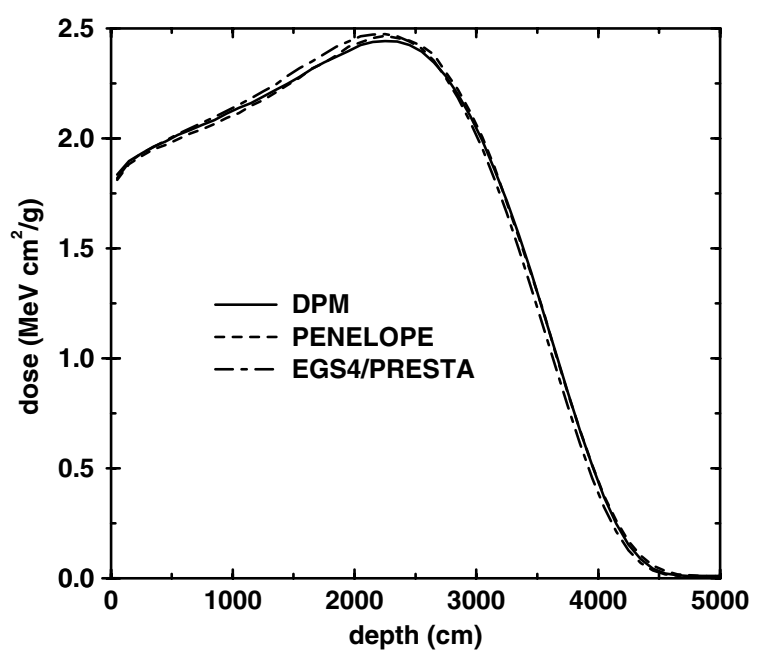

Figure 8. Depth dose for a $10 \mathrm{MeV}$ normal pencil beam in an air phantom.

hard inelastic interaction as if the MFP were independent of energy along the path up to the interaction point. This approximation works well for Møller interactions due to the relatively slow variation of their MFP over the relevant energy range, as reflected in equation (25). Although still acceptable, it does not work equally well for bremsstrahlung, equation (31). Therefore a small error is introduced at those energies for which the bremsstrahlung contribution to the dose is not negligible. Since the slope of the bremsstrahlung MFP as a function of the electron energy is negative in the region of interest, DPM systematically underestimates this MFP. This effect is equivalent to an overestimation of the radiative stopping power, which will appear as an increase in the dose at shallow depths when it dominates over other sources of error.

In figures 10 and 11 depth dose curves in water for 15 and $20 \mathrm{MeV}$ beams are represented to show the increase of the overestimation of the radiative stopping power as the energy of the 


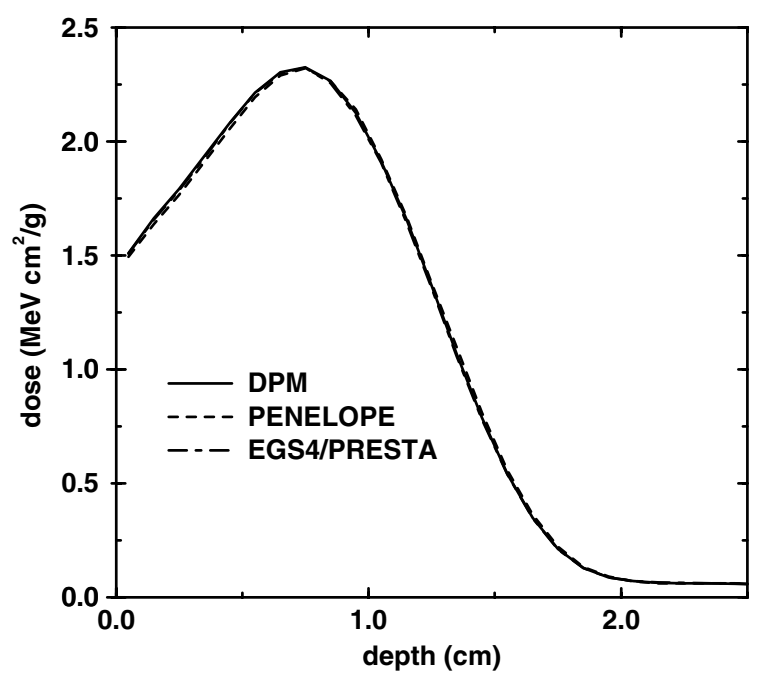

Figure 9. Depth dose for a $15 \mathrm{MeV}$ normal pencil beam in a titanium phantom.

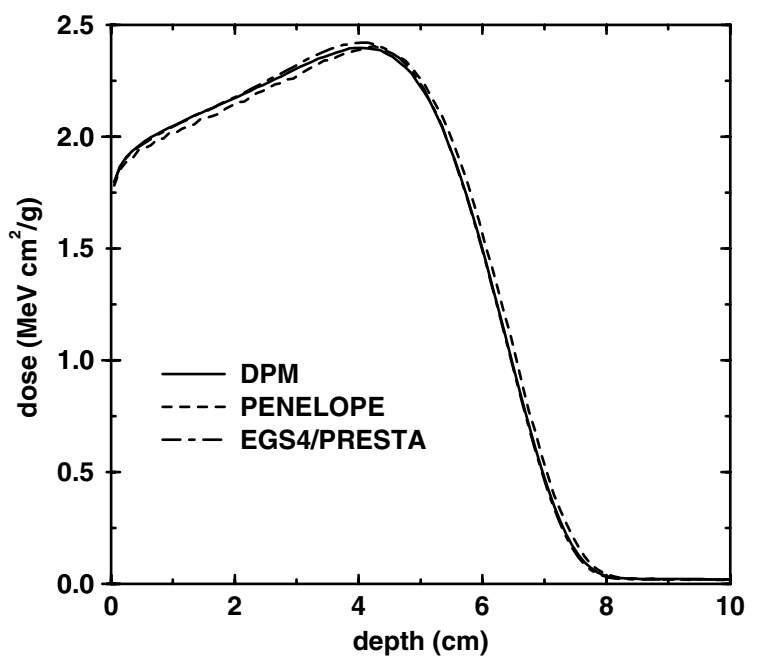

Figure 10. Depth dose for a $15 \mathrm{MeV}$ pencil beam impinging on water.

beam increases. Despite the approximation on the bremsstrahlung cross section, the agreement is good and no correction for this effect is needed below $20 \mathrm{MeV}$.

Figure 12 shows the effect of these approximations in an extreme case, that is, for a very high- $Z$ material and at the highest energy considered. With the current DPM model, discrepancies of up to $8 \%$ are seen between DPM and other MC programs. By switching to a method in which energy loss between collisions is accounted for in updating $t_{\mathrm{B}}$, the difference between DPM and both PENELOPE and EGS4 can be reduced to 3-5\%, as seen in the figure. However, as this introduces a computational overhead of close to $10 \%$ because of the frequency with which the cross sections must be computed and this effect is significant only for thick targets and very high $Z$, this correction is not retained in the basic DPM model. 


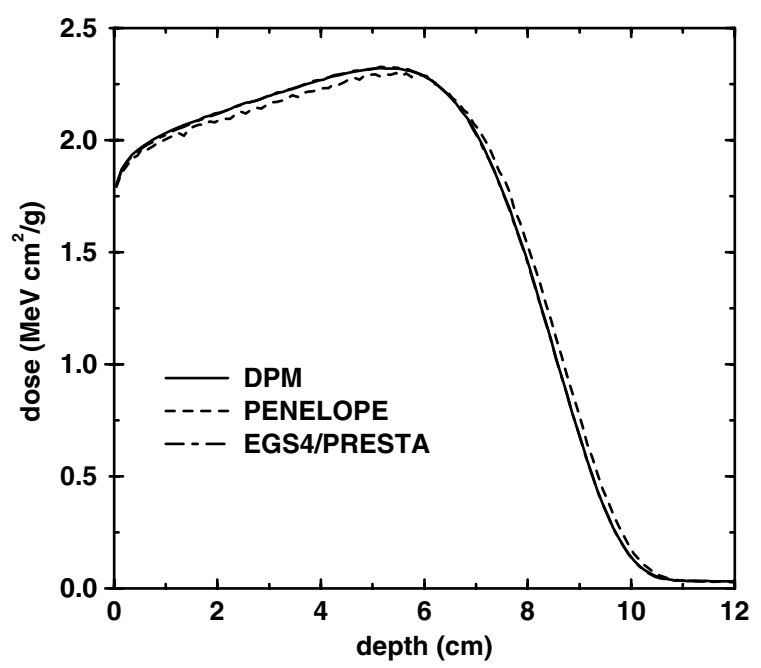

Figure 11. Depth dose for a $20 \mathrm{MeV}$ pencil beam impinging on water.

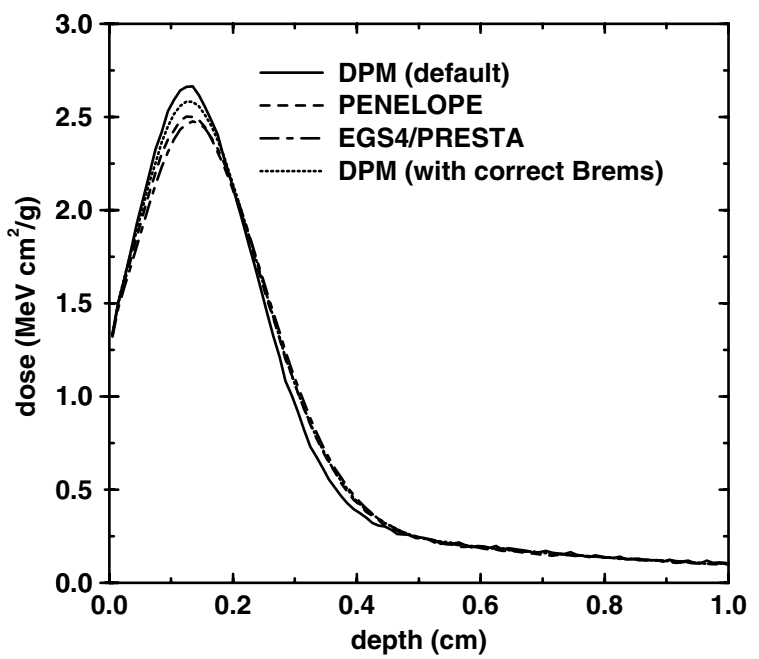

Figure 12. Depth dose produced by a $20 \mathrm{MeV}$ pencil beam on a tungsten $(Z=74)$ phantom.

\subsection{Inhomogeneities}

Several multiple slab configurations were chosen to test DPM with inhomogeneous geometries, and results are presented in figures 13 to 15 . Good agreement is found between the results from the three codes, with differences of the order of $1 \%$ of the dose maximum, reaching a maximum of 2\% between PENELOPE and EGS4 or DPM in the higher-energy cases.

\subsection{CT geometry}

We present below results of dose computations using representative CT data to model density variation in a voxelized geometry. As the default PENELOPE package was designed to work with objects made of solid bodies with constant densities, it is unable to handle the density 


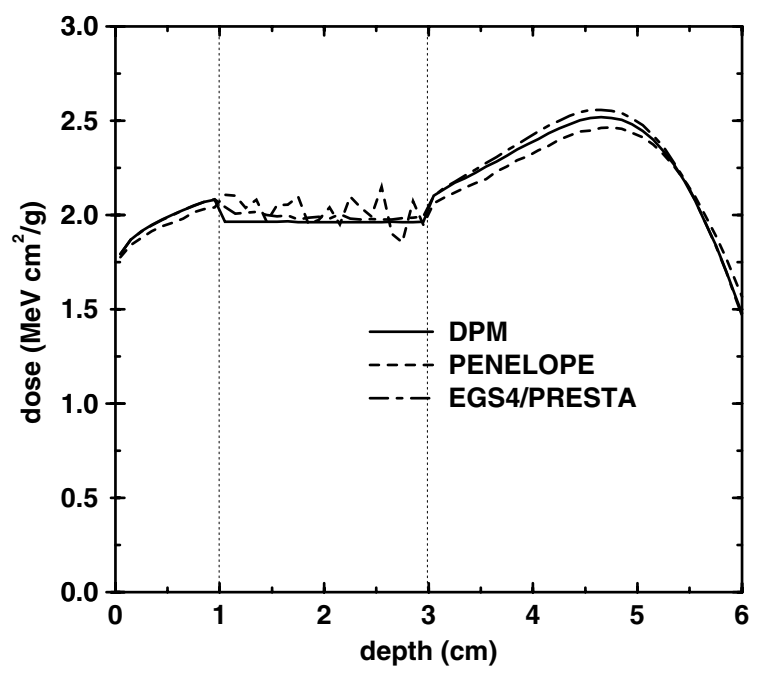

Figure 13. Depth dose in a water phantom with an air layer. The beam energy was $10 \mathrm{MeV}$.

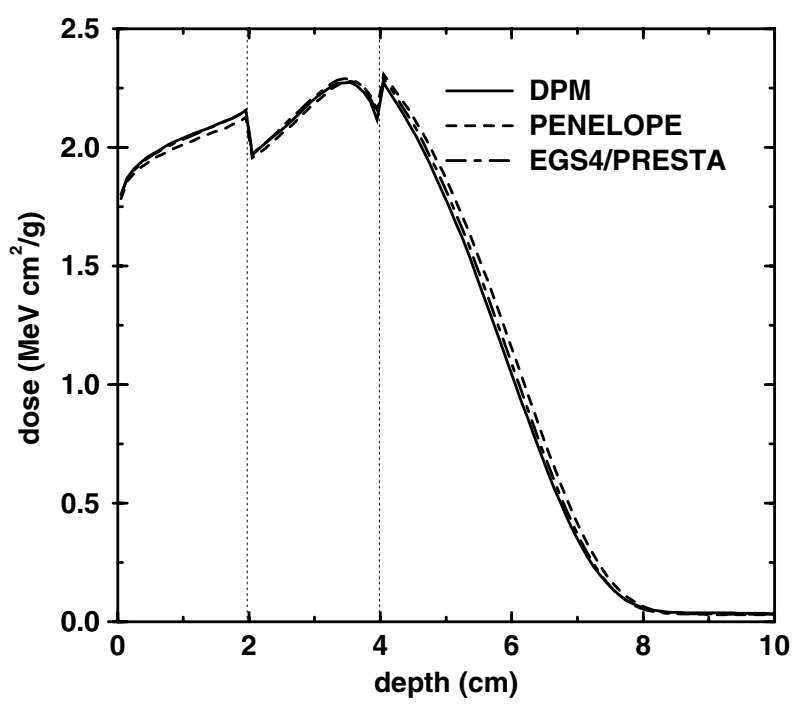

Figure 14. Depth dose in a water phantom with a bone layer. The beam energy was $18 \mathrm{MeV}$.

variations between neighbouring voxels present in the geometry of a real patient $\dagger$. A utility for modelling density variations between regions does exist in the EGS4 system, and so this feature was exploited in generating simulation results for comparison with DPM.

In figure 16 a slice of the patient scan used for the current simulations is shown. Results from these calculations, which assumed a fictitious $16 \mathrm{MeV}$ electron beam, are presented in figures 17 to 19 . In order to facilitate comparisons of doses for individual voxels, a very large number of histories $\left(10^{9}\right)$ were simulated. This number is orders of magnitude higher than that required for a routine treatment plan simulation, and gives a standard deviation of approximately $0.3 \%$ of the dose maximum for voxels of $1 \mathrm{~mm}$ on a side. The agreement

$\dagger$ A voxel based version of PENELOPE is currently being developed. 


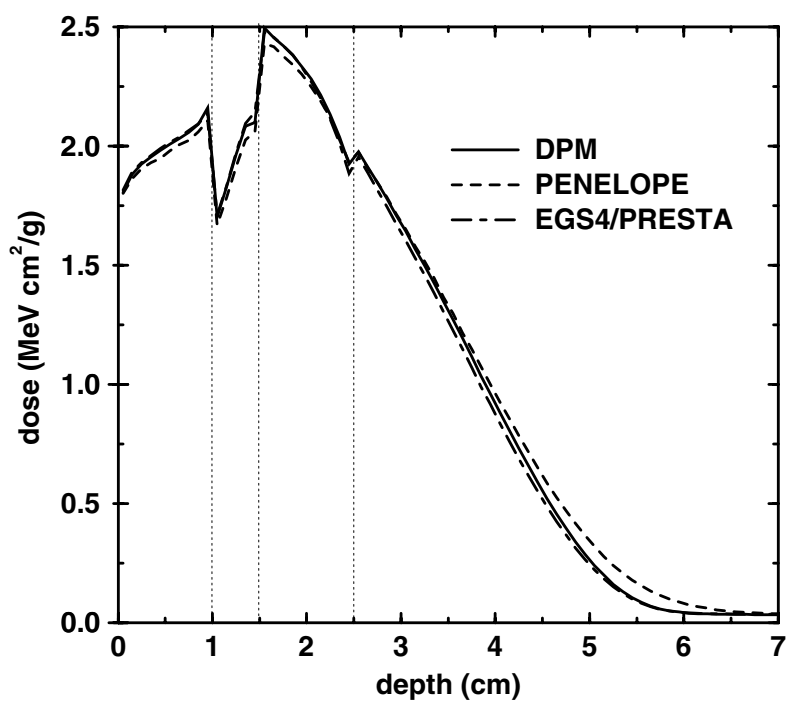

Figure 15. Depth dose in a multilayered water/titanium/bone/water geometry. The beam energy was $15 \mathrm{MeV}$.

between DPM and EGS4 is good, as expected from the previous results for homogeneous and multilayered geometries. The differences in the voxel doses are less than $3 \%$ of the overall maximum dose found in the geometry. If the differences are referred to the maximum dose in each figure, only the case with the lowest dose $(x=28.5 \mathrm{~mm})$ significantly exceeds $3 \%$ of that maximum, ranging from 3 to $8 \%$. Moreover, dose volume histograms (DVHs) were obtained for a specific target volume (or region of interest, ROI) of the same CT shown in figure 16. In figure 20 these DVHs are compared, showing an excellent agreement, with differences of the order of a few per cent.

\section{Timing and efficiency}

In table 1 we present the measured CPU times required to run 1 million histories on several of the test problems reported earlier. All runs were performed on an HP C3000 workstation, which is based on a $400 \mathrm{MHz}$ HP PA8500 CPU. The program was compiled with the HP-UX f77 compiler and the recommended optimization switches +04 +E1 +E4 +E6 - K +U77. The reference step size was chosen to be the largest such that the DPM results lay within $1 \%$ of the EGS4 results. This value was $0.5 \mathrm{~cm}$ in all cases except for $5 \mathrm{MeV}$ on water, for which it was necessary to use a $0.2 \mathrm{~cm}$ step. Note that the $16 \mathrm{MeV} \mathrm{CT}$ case requires more time than the $20 \mathrm{MeV}$ water case because the envelope of air and other less dense media under the beam give rise to deeper penetration of the source particles, requiring more computations of energy deposition and voxel crossings.

Table 2 presents results from a profiling study of DPM. Values are expressed in percentage of overall CPU time spent in various process. Two important conclusions can be drawn. First, the time taken in multiple scattering processes is quite small $(\sim 3 \%)$, implying that little further speed-up can be achieved in electron transport Monte Carlo simulations through manipulating step sizes. Second, a great deal of time (41\%) is spent in voxel-to-voxel boundary crossings and CSDA energy deposition calculations, two unavoidable tasks of any algorithm based on mixed simulation of the inelastic interactions. Therefore, DPM can be considered 


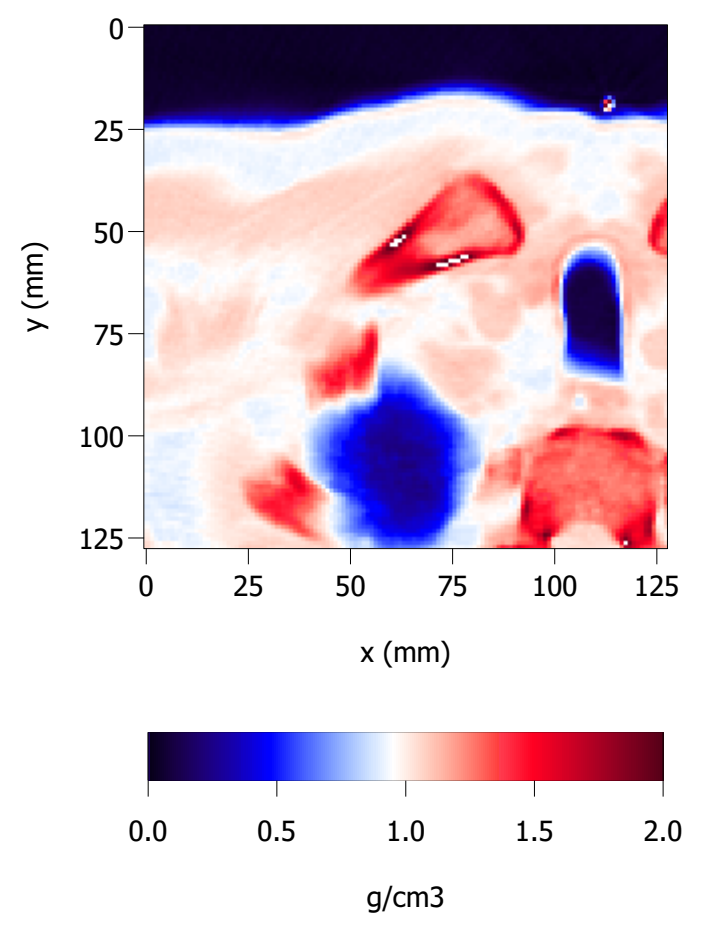

Figure 16. CT image representing a slice located $5.5 \mathrm{~cm}$ deep in the $z$ direction, perpendicular to the paper. The $x$-axis goes from left to right and the $y$-axis points downwards. The 'universe' of the simulation consists in $128 \times 128 \times 128$ cubic voxels of side $1 \mathrm{~mm}$. A fictitious $16 \mathrm{MeV}$ electron beam coming along the positive direction of the $y$-axis was defined, entering the universe through a $5 \times 5 \mathrm{~cm}^{2}$ square covering the range $(x, z)=(3.9-8.9,3.9-8.9) \mathrm{cm} .10^{9}$ histories were simulated to obtain the results presented in the next figures.

(This figure is in colour only in the electronic version, see www.iop.org)

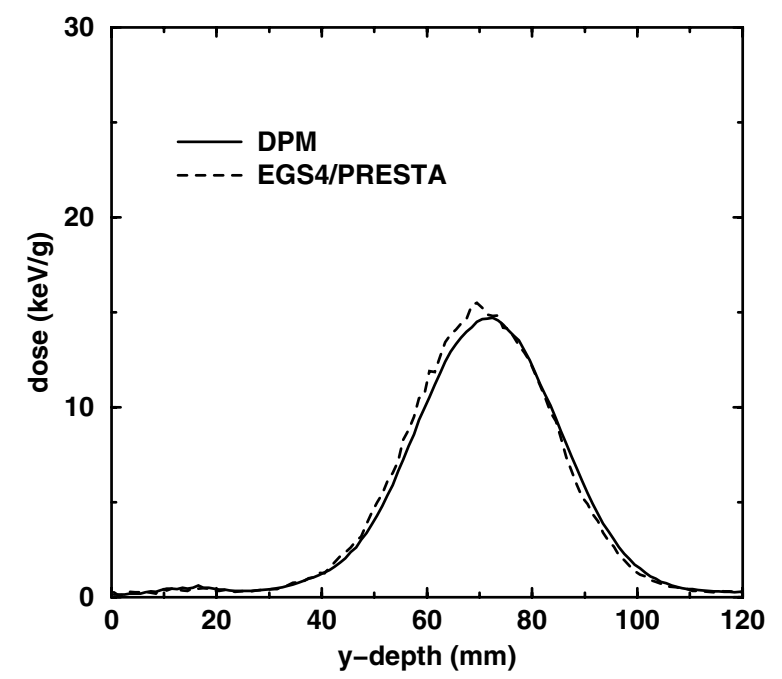

Figure 17. Dose along a line parallel to the $y$-axis of the CT slice represented in figure 16 at the value of $x=28.5 \mathrm{~mm}$. Notice that the considered voxels lay outside the source field. 


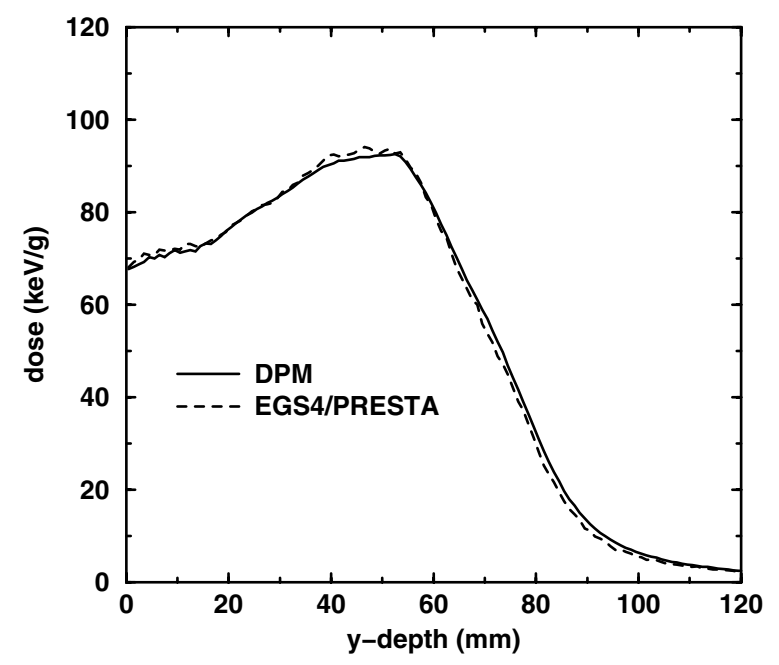

Figure 18. Dose along a line parallel to the $y$-axis of the CT slice represented in figure 16 at the value of $x=60.5 \mathrm{~mm}$. Notice that the considered voxels lay directly under the source field.

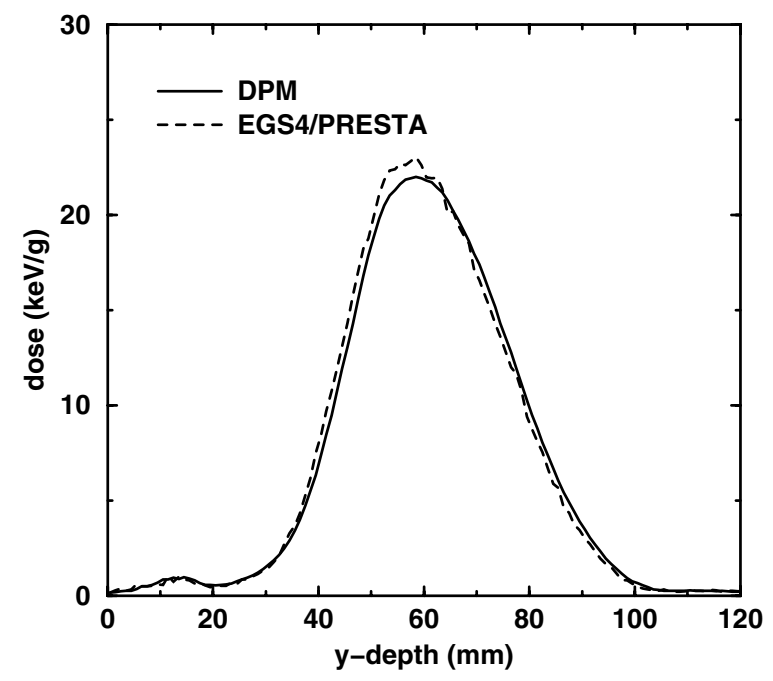

Figure 19. Dose along a line parallel to the $y$-axis of the CT slice represented in figure 16 at the value of $x=94.5 \mathrm{~mm}$. The considered voxels lay outside the source field.

to exhibit close to the maximum achievable efficiency for condensed history CSDA MC codes.

\section{Conclusion}

A fast MC algorithm for the simulation of the dose deposited by electron-photon showers under radiotherapy conditions has been developed. DPM takes advantage of a new transport mechanics and an accurate multiple-scattering formalism independent of $Z$, permitting long simulation steps across media boundaries, significantly increasing the efficiency of the 


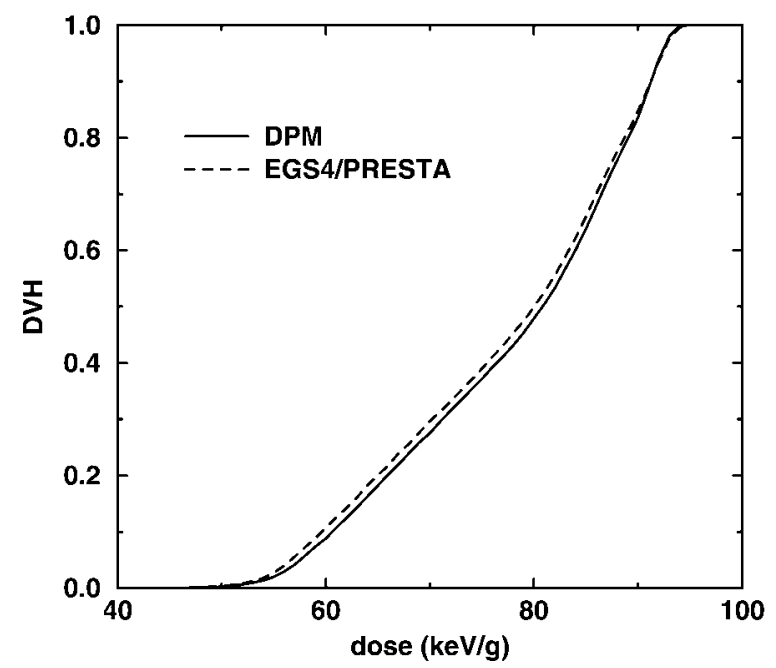

Figure 20. Integral DVHs obtained for the ROI defined by a cube of $2 \times 2 \times 2 \mathrm{~cm}^{3}$ centred at $x=6.4, y=5.9$ and $z=5.5 \mathrm{~cm}$ of the CT shown in figure 16 .

Table 1. CPU time for one million histories.

\begin{tabular}{lll}
\hline Figure & Problem description & CPU time (s) \\
\hline 5 & $10 \mathrm{MeV}$ pencil beam on water slab & 169.4 \\
6 & $10 \mathrm{MeV}$ broad beam on water slab & 181.4 \\
4 & $5 \mathrm{MeV}$ pencil beam on water slab & 111.2 \\
10 & $15 \mathrm{MeV}$ pencil beam on water slab & 250.6 \\
11 & 20 MeV pencil beam on water slab & 327.0 \\
$17-20$ & $16 \mathrm{MeV}$ broad beam on CT geometry & 383.2 \\
\hline
\end{tabular}

Table 2. Code profile. Use of $\mathrm{CPU}$ time in per cent for $16 \mathrm{MeV}$ electrons incident on CT profile phantom.

\begin{tabular}{|c|c|c|}
\hline \multirow[t]{2}{*}{$86 \mathrm{e}^{-}$transport } & $\begin{array}{l}= \\
55 \text { transport through voxels }\end{array}$ & $\begin{array}{l}= \\
14 \text { geometry handling } \\
41 \text { CSDA } E \text { loss and translation }\end{array}$ \\
\hline & $\begin{array}{l}3 \text { transport to collisions } \\
3 \text { sample scattering \& rotations } \\
25 \text { data table look-ups }\end{array}$ & \\
\hline \multicolumn{3}{|l|}{2 photon transport } \\
\hline \multicolumn{3}{|l|}{$1 \mathrm{I} / \mathrm{O}$} \\
\hline 11 tallying & & \\
\hline
\end{tabular}

computation without appreciably distorting the results. DPM has been shown to reproduce the dose distributions calculated with high-accuracy state-of-the-art general-purpose MC codes within an error of the order of $1.25 \%$ of the dose maximum, but with significant increase in computational efficiency. Dosimetric results accurate enough for electron beam radiotherapy applications can be generated in times of the order of 5 min on desktop workstations. 
It has been pointed out (Bielajew 1994a, 1997) that present radiotherapy treatment planning systems, based on some type of analytic approximation to the solution of the transport equation will, some day, be replaced by systems based on the much more accurate and conceptually simpler MC methods. This work has shown that the use of these methods for both fast and accurate simulation of the transport of electrons in CT geometries is indeed feasible.

\section{Acknowledgments}

We would like to thank Drs Dick Fraass, Kwok Lam, Dan McShan and Randy Ten Haken, from the Radiation Oncology department of the University of Michigan, Dr Ed Larsen from the Department of Nuclear Engineering and Radiological Sciences, and Dr F Salvat, from the ECM Department of the Universitat de Barcelona, for fruitful discussions. One of the authors (JS) gratefully acknowledges the financial support of the Direcció General de Recerca de la Generalitat de Catalunya (Spain) grant no 1997BEAI400256 as well as the financial support from the Spanish Fondo de Investigación Sanitaria under contract no 98/0047-01. Financial support for this work has also been partially provided under the auspices of the US Department of Energy by the Lawrence Livermore National Laboratory under contract number W-7405ENG-48, and by ADAC Laboratories (Milpiltas, CA).

\section{References}

Andreo P 1991 Monte Carlo techniques in medical radiation physics Phys. Med. Biol. 36 861-920

Baró J, Sempau J, Fernández-Varea J M and Salvat F 1995 PENELOPE: an algorithm for Monte Carlo simulation of the penetration and energy loss of electrons and positrons in matter Nucl. Instrum. Methods B 100 31-46

Berger M J 1963 Monte Carlo calculation of the penetration and diffusion of fast charged particles Methods Comput. Phys. 1 135-215

Bethe H A 1953 Molière's theory of multiple scattering Phys. Rev. 89 1256-66

Bielajew A F 1994a Monte Carlo modelling in external electron-beam radiotherapy-why leave it to chance? Proc. of the 11th Conf. on the Use of Computers in Radiotherapy (Madison, WI: Medical Physics Publishing) pp 2-5

-1994b Plural and multiple small-angle scattering from a screened Rutherford cross section Nucl. Instrum. Methods B 86 257-269

1997 Monte Carlo dose calculation: why gamble with anything else? Proc. of the First International Workshop on EGS4 (Tsukuba City, Japan: Laboratory for High Energy Physics) pp 310-23

Bielajew A F, Hirayama H, Nelson W R and Rogers D W O 1994 History, overview and recent improvements of EGS4 National Research Council of Canada Report PIRS-0436

Bielajew A F and Rogers D W O 1989 Variance-reduction techniques Monte Carlo Transport of Electrons and Photons ed T Jenkins et al (New York: Plenum) pp 407-19

Bielajew A F and Salvat F 2000 Improved electron transport mechanics in the PENELOPE Monte Carlo model Nucl. Instrum. Methods submitted

Bothe W 1921a Das allgemeine Fehlergesetz, die Schwankungen der Feldstärke in einem Dielektrikum und die Zerstreuung der $\alpha$-Strahlen Z. Phys. 5 63-9

—1921b Die Gültigkeitsgrenzen des Gaußschen Fehlergesetzes für unabhängige Elementarfehlerquellen Z. Phys. 4 161-77

Briesmeister J F 1993 MCNP - a general Monte Carlo N-particle transport code Los Alamos National Laboratory Report LA-12625-M (Los Alamos, NM)

Cygler J, Battista J J, Scrimger J W, Mah E and Antolak J 1987 Electron dose distributions in experimental phantoms: a comparison with 2D pencil beam calculations Phys. Med. Biol. 32 1073-86

Fernández-Varea J M, Mayol R, Baró J and Salvat F 1993 On the theory and simulation of multiple elastic scattering of electrons Nucl. Instrum. Methods B73 447-73

Goudsmit S A and Saunderson J L 1940a Multiple scattering of electrons Phys. Rev. 57 24-9

1940b Multiple scattering of electrons. II Phys. Rev. 58 36-42

Halbleib J 1989 Structure and operation of the ITS code system Monte Carlo Transport of Electrons and Photons ed T Jenkins et al (New York: Plenum) pp 249-62 
Halbleib J A, Kensek R P, Mehlhorn T A, Valdez G D, Seltzer S M and Berger M J 1992 ITS Version 3.0: the integrated TIGER series of coupled electron/photon Monte Carlo transport codes Sandia Report SAND91-1634

Hartmann-Siantar C L et al 1997 Lawrence Livermore National Laboratory's PEREGRINE project Proc. of the 12th Conf. on the Use of Computers in Radiotherapy (Madison, WI: Medical Physics Publishing) pp 19-22

Hartmann-Siantar C L, Chandler W P, Rathkopf J A, Svatos M M and White R M 1995 PEREGRINE: an all-particle Monte Carlo code for radiation therapy Proc. Int. Conf. on Mathematics and Computations, Reactor Physics and Environmental Analyses (La Grange Park, IL: American Nuclear Society Press) pp 857-65

Heitler W 1954 The Quantum Theory of Radiation (Oxford: Clarendon)

Kawrakow I and Bielajew A F 1998a On the condensed history technique for electron transport Nucl. Instrum. Methods B 142 253-80

-1998b On the representation of electron multiple elastic-scattering distributions for Monte Carlo calculations Nucl. Instrum. Methods B 134 325-36

Kawrakow I, Fippel M and Friedrich K 1996 3D electron dose calculation using a voxel based Monte Carlo algorithm Med. Phys. 23 445-57

Keall P J and Hoban P W 1996 Super-Monte Carlo: a 3D electron beam dose calculation algorithm Med. Phys. 23 2023-34

Knöös T, Nilsson M and Ahlgren L 1986 A method for conversion of Hounsfield number to electron density and prediction of macroscopic pair production cross-sections Radiother. Oncol. 5 337-45

Larsen E W 1992 A theoretical derivation of the condensed history algorithm Ann. Nucl. Energy 19 701-14

Lewis H W 1950 Multiple scattering in an infinite medium Phys. Rev. 78 526-9

Ma C M, Mok E, Kapur A, Findley D, Brain S and Boyer A L 1999 Clinical implementation of a Monte Carlo treatment planning system Med. Phys. 26 2133-43

Ma C M, Reckwerdt P, Holmes M, Rogers D W O and Geiser B 1995 DOSXYZ users manual NRC Report PIRS 509b

Mayol R and Salvat F 1997 Total and transport cross sections for elastic scattering of electrons by atoms At. Data Nucl. Data Tables 65 55-154

Mohan R 1997 Why Monte Carlo? Proceedings of the 12th Conference on the Use of Computers in Radiotherapy (Madison, WI: Medical Physics Publishing) pp 16-18

Molière G Z 1947 Theorie der Streuung schneller geladener Teilchen. I. Einzelstreuung am abgeschirmten CoulombField Z. Naturforsch. 2a 133-45

_ 1948 Theorie der Streuung schneller geladener Teilchen. II. Mehrfach- und Vielfachstreuung Z. Naturforsch. 3a $78-97$

Nelson W R, Hirayama H and Rogers D W O 1985 The EGS4 code system Stanford Linear Accelerator Center Report SLAC-265

Neuenschwander H and Born E J 1992 A Macro Monte Carlo method for electron beam dose calculations Phys. Med. Biol. 37 107-25

Neuenschwander H, Mackie T R and Reckwerdt P J 1995 MMC—A high-performance Monte Carlo code for electron beam treatment planning Phys. Med. Biol. 40 543-74

Ribberfors R 1975 Relationship of the relativistic Compton cross section to the momentum distribution of bound electron states Phys. Rev. B 12 2067-74

Rogers D W O and Bielajew A F 1989a A comparison of EGS and ETRAN Monte Carlo Transport of Electrons and Photons ed T Jenkins et al (New York: Plenum) pp 323-44

- 1989b Experimental benchmarks of EGS Monte Carlo Transport of Electrons and Photons ed T Jenkins et al (New York: Plenum) pp 307-22

1990 Monte Carlo techniques of electron and photon transport for radiation dosimetry The Dosimetry of Ionizing Radiation vol III, ed K Kase, B Bjärngard and F Attix (New York: Academic)

Rogers D W O, Ma C M, Ding G X and Walters B 1995 BEAM users manual NRC Report PIRS 509a

Salvat F and Fernández-Varea J M 1992 Semiempirical cross sections for the simulation of the energy loss of electrons and positrons in matter Nucl. Instrum. Methods B 63 255-69

Salvat F, Fernández-Varea J M, Baró J and Sempau J 1996 PENELOPE, an algorithm and computer code for Monte Carlo simulation of electron-photon showers Ciemat (Centro de Investigaciones Energéticas, Medioambientales y Tecnológicas) Technical Report no 799

Seltzer S M 1989 An overview of ETRAN Monte Carlo methods Monte Carlo Transport of Electrons and Photons ed T Jenkins et al (New York: Plenum) pp 153-82

1991 Electron-photon Monte Carlo calculations: the ETRAN code Int. J. Appl. Radiat. Isotopes 42 917-41

Seltzer S M and Berger M J 1985 Bremsstrahlung spectra from electron interactions with screened atomic nuclei and orbital electrons Nucl. Instrum. Methods Phys. Res. B 12 95-134

Sempau J, Acosta E, Baró J, Fernández-Varea J M and Salvat F 1997 An algorithm for Monte Carlo simulation of coupled electron-photon showers Nucl. Instrum. Methods B 132 377-390 
Wentzel G 1922 Zur theorie der streuung von $\beta$-strahlen Ann. Phys. 69 335-68

Woodcock E, Murphy T, Hemmings P and Longworth S 1965 Techniques used in the GEM code for Monte Carlo neutronics calculations in reactors and other systems of complex geometry Proc. Conf. on Applications of Computing Methods to Reactor Problems Argonne National Laboratories Report ANL-7050 